\title{
GALERKIN METHODS FOR A SCHRÖDINGER-TYPE EQUATION WITH A DYNAMICAL BOUNDARY CONDITION IN TWO DIMENSIONS *
}

\author{
D.C. Antonopoulou
}

\begin{abstract}
In this paper, we consider a two-dimensional Schrödinger-type equation with a dynamical boundary condition. This model describes the long-range sound propagation in naval environments of variable rigid bottom topography. Our choice for a regular enough finite element approximation is motivated by the dynamical condition and therefore, consists of a cubic splines implicit Galerkin method in space. Furthermore, we apply a Crank-Nicolson time stepping for the evolutionary variable. We prove existence and stability of the semidiscrete and fully discrete solution. Due to the complexity of the analyzed problem, we use very refined technics in order to derive estimates of the numerical error in the $H^{1}$-norm.
\end{abstract}

Mathematics Subject Classification. 65M60, 65M12, 65M15, 76Q05.

Received December 1, 2013. Revised July 31, 2014.

Published online June 30, 2015.

\section{INTRODUCTION}

\subsection{The 'Parabolic' equation with Neumann bottom boundary condition}

The Helmholtz equation is a model for the long-range sound propagation in the sea that observes the acoustic pressure as a function of depth, range - which is the horizontal distance from the acoustic source, and azimuth. In cylindrical coordinates, this equation takes the form

$$
p_{r r}+\frac{1}{r} p_{r}+\frac{1}{r^{2}} p_{\vartheta \vartheta}+p_{x x}+k_{0}^{2} n_{\mathrm{ref}}^{2} p=0,
$$

where $p$ is the acoustic pressure. The variable $r$ in $\left[R_{0}, R\right]$ is the horizontal distance from a harmonic point source which is placed on the $x$ axis emitting at a frequency $f_{0}$. The depth variable $x \geq 0$ is increasing downwards and $\vartheta$ is the azimuth varying in the interval $\left[\vartheta_{1}, \vartheta_{2}\right]$ with $0 \leq \vartheta_{1}<\vartheta_{2} \leq 2 \pi$. Furthermore,

$$
k_{0}:=\frac{2 \pi f_{0}}{c_{0}}
$$

Keywords and phrases. 2-D Schrödinger equation, finite element methods, error estimates, noncylindrical domain, Neumann boundary condition, cubic splines, Crank-Nicolson time stepping, dynamical boundary condition, underwater acoustics.

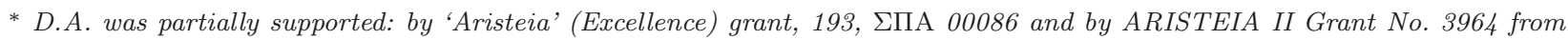
the General Secretariat of Research and Technology, Greece.

1 Department of Mathematics, University of Chester, Thornton Science Park, CH2 4NU, UK. d.antonopoulou@chester.ac.uk 
is a reference wave number where the constant $c_{0}$ is a reference sound speed, and

$$
n_{\mathrm{ref}}(r, x, \vartheta):=\frac{c_{0}}{c(r, x, \vartheta)},
$$

is the refraction index, where $c(r, x, \vartheta)$ is the sound speed in the water. The medium is inhomogeneous, therefore, the refraction index depends on all variables.

The application of an envelop transformation and a paraxial far-field approximation lead to the standard narrow-angle 2D 'Parabolic' Equation (PE), where '2D' refers to the space variables, $[15,17,18]$. (PE) is the following Schrödinger-type equation

$$
\Psi_{r}=\frac{\mathrm{i}}{2 k_{0}}\left(\Psi_{x x}+\frac{1}{r^{2}} \Psi_{\vartheta \vartheta}\right)+\mathrm{i} \frac{k_{0}}{2}\left(n_{\text {ref }}^{2}-1\right) \Psi .
$$

The unknown function $\Psi$ stands as a measure for the acoustic pressure in inhomogeneous, weakly rangedependent marine environments.

More specifically, the envelop transformation is

$$
p(r, x, \vartheta)=\frac{\Psi(r, x, \vartheta) e^{\mathrm{i} k_{0} r}}{\sqrt{k_{0} r}} .
$$

Then, the equation (1.1) is derived under the assumptions of a paraxial approximation, i.e. that

$$
\left|2 \mathrm{i} k_{0} \Psi_{r}\right|>>\left|\Psi_{r r}\right|
$$

and of the far field approximation

$$
k_{0} r>>1,
$$

which means that $(\mathrm{PE})$, as an approximate model, describes the sound transmission very far from the acoustic source.

This approximation is motivated by the fact that Helmholtz equation is very difficult to be analyzed mathematically while its numerical analysis is highly nontrivial, since it is of elliptic type and is posed on a 3 -dimensional domain. Simulations for realistic marine environments of range $R$ equal to some kilometers and of medium depth, easily result in linear systems where the number of unknowns is of order $\mathcal{O}\left(10^{6}\right)$; this numerical cost restricts significantly the use of this equation in practice. (PE), being an evolutionary equation of Schrödinger type, is much more accessible.

We assume that the variable bottom topography in cylindrical coordinates is given by a positive surface $x=s(r, \vartheta)$. The equation (1.1) is posed on the noncylindrical domain ( $c f$. Fig. 1),

$$
\mathbb{S}:=\left\{(r, x, \vartheta): r \in\left[R_{0}, R\right], \vartheta \in\left[\vartheta_{1}, \vartheta_{2}\right], x \in[0, s(r, \vartheta)]\right\},
$$

where, of course, the boundary values of space variables depend on the evolutionary variable $r$. At a given distance $r$, these values generate a union of 4 curves which are embedded in $\mathbb{R}^{3}$ given as $\bigcup_{i=1}^{4} S_{i}(r)$, for:

$$
\begin{gathered}
S_{1}(r):=\left\{(r, 0, \vartheta): \vartheta \in\left[\vartheta_{1}, \vartheta_{2}\right]\right\}, \\
S_{2}(r):=\left\{\left(r, x, \vartheta_{1}\right): x \in\left[0, s\left(r, \vartheta_{1}\right)\right]\right\}, \\
S_{3}(r):=\left\{(r, s(r, \vartheta), \vartheta): \vartheta \in\left[\vartheta_{1}, \vartheta_{2}\right]\right\} \quad \text { (the variable boundary), }
\end{gathered}
$$

and

$$
S_{4}(r):=\left\{\left(r, x, \vartheta_{2}\right): x \in\left[0, s\left(r, \vartheta_{2}\right)\right]\right\}
$$

$c f$. Figure 2. 


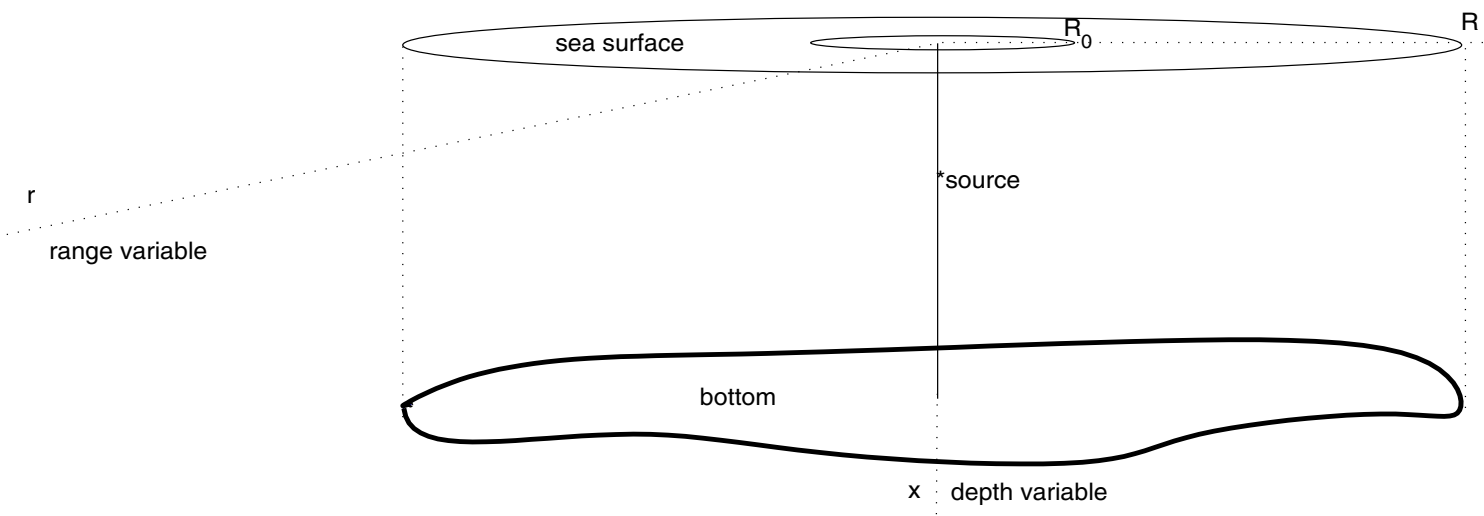

Figure 1. The naval environment.

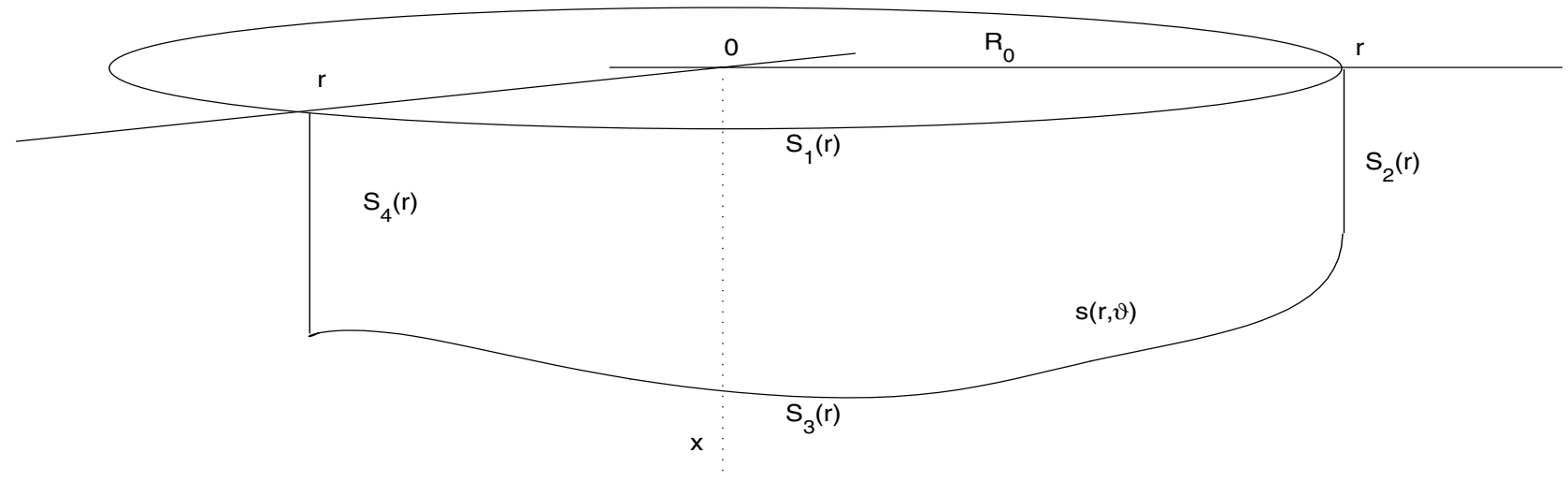

Figure 2. The boundary at a fixed distance $r$.

The initial condition for the equation (1.1) is given by

$$
\Psi\left(R_{0}, x, \vartheta\right)=\Psi_{0}(x, \vartheta) \quad \forall \vartheta \in\left[\vartheta_{1}, \vartheta_{2}\right], \forall x \in\left[0, s\left(R_{0}, \vartheta\right)\right],
$$

where $\Psi_{0}$ represents a harmonic point source and is usually defined as a smooth function with compact support, for example as a linear combination of Gaussian starters, cf. [18]. The horizontal surface of the domain which, for given $r$, refers to $S_{1}(r)$ (sea surface) and the lateral boundaries $S_{2}(r)$ and $S_{4}(r)$ are assumed to be perfectly absorbing which is mathematically modeled by imposing the following homogeneous Dirichlet boundary condition

$$
\Psi(r, x, \vartheta)=0 \quad \text { on } \quad S_{1}(r) \cup S_{2}(r) \cup S_{4}(r) .
$$

In addition, a Neumann condition modeling the acoustically rigid bottom is posed along the variable boundary $S_{3}(r)$, i.e.

$$
\frac{\partial \Psi}{\partial n}(r, \cdot)=0 \quad \text { on } \quad S_{3}(r)
$$

which yields, since the domain is noncylindrical

$$
\Psi_{x}(r, x, \vartheta)-\frac{1}{r^{2}} s_{\vartheta}(r, \vartheta) \Psi_{\vartheta}(r, x, \vartheta)=s_{r}(r, \vartheta) \Psi_{r}(r, x, \vartheta) \quad \text { on } \quad S_{3}(r) .
$$




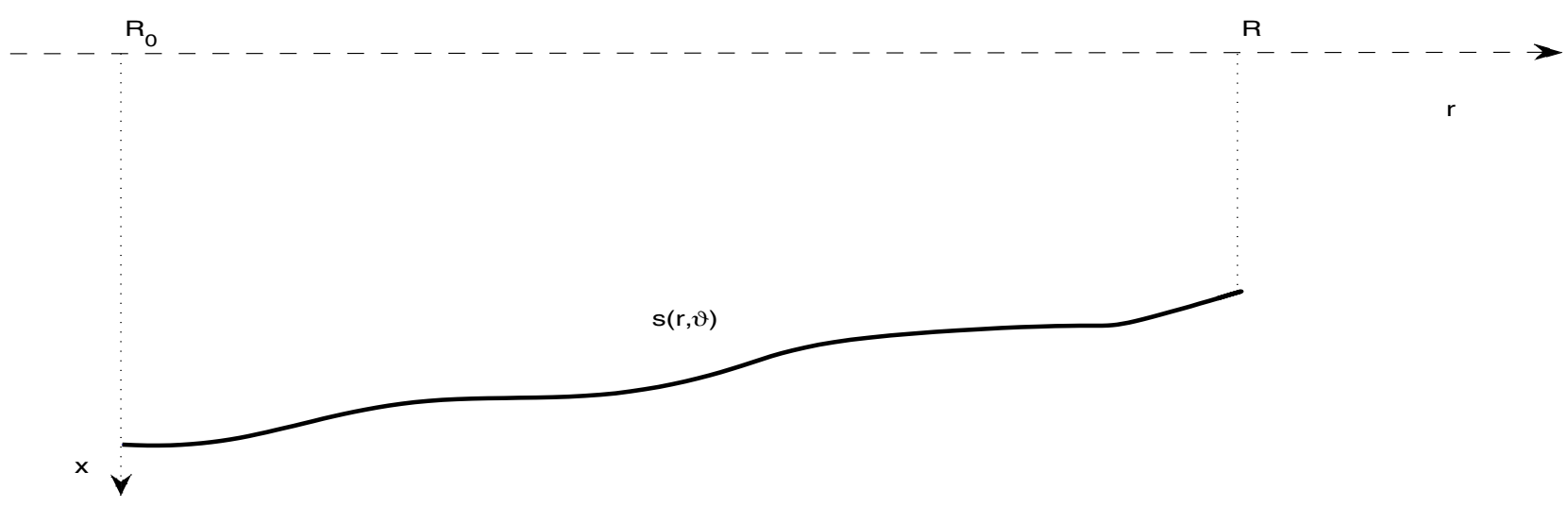

Figure 3. Bottom profile at a fixed azimuth $\vartheta$.

This boundary condition is a nonstandard dynamical one because it includes the term $\Psi_{r}$ and $r$ is the time-like evolutionary variable; note that $r$ corresponds to time in the usual Schrödinger equation of quantum mechanics.

The equation so-obtained is of Schrödinger type, with Dirichlet condition for the free surface on the top and Neumann condition along the bottom. The mathematical analysis of this problem is intricate when the profile of the bottom varies with the distance $r$. Depending on the sign of term $s_{r}$ which is introduced in the leading term of the Neumann condition, certain difficulties may be encountered.

In the axial symmetric case, which translates to an one-dimensional problem where the bottom $s$ is only a function of range $r$, Abrahamsson and Kreiss proved well-posedness when $s$ is strictly monotone, cf. $[1,2]$. Further, they observed the development of a significant amplitude increase in the long range propagation for certain downsloping bottom profiles $\left(s_{r} \geq 0\right)$. The analogous results were presented in [8], by means of numerical simulations with finite element methods, for various cases of downsloping bottoms; in fact, numerical blow-up was observed for such bottoms in the presence of inflection points. Remind that $s_{r}$ is the coefficient of the dynamical term $\Psi_{r}$, the sign of which seems to be important for the Heat equation with dynamical conditions also, $c f .[13,19]$ for the theoretical p.d.e. problem and [8] for the optimal order error analysis of the constructed numerical scheme.

In this paper we will apply Galerkin finite element methods to the initial and boundary problem (1.1)-(1.4), when $s$ is smooth, and decreasing in range (upsloping bottom), more specifically under the following assumptions holding on the domain of definition of s (cf. Fig. 3):

$$
s_{r}(r, \vartheta) \leq 0 \text { for any } r, \vartheta \text {, where if } s_{r}\left(r_{0}, \vartheta_{0}\right)=0 \text { for some } r_{0}, \vartheta_{0} \text {, then } s_{\vartheta}\left(r_{0}, \vartheta_{0}\right)=0 \text { also. }
$$

Obviously, a strictly monotone topography $\left(s_{r}<0\right)$ satisfies $(1.5)$. In addition, this relation is in general valid for monotone bottom profiles $\left(s_{r} \leq 0\right)$ where critical points in distance may appear, under the restriction though that any of these points must be a critical point in azimuth also.

The problem analyzed, posed in a single layer of water over a bottom of variable topography, is an idealized one. More realistic environments consist of a layer of water above several layers of fluid sediments, $c f$. [11] for a review considering the problem with interfaces. Furthermore, one has to take into account the shear stress yielding the so-called elastic (TBC) conditions, [12].

Considering the one-dimensional problem posed on a variable domain there exists an intensive rigorous numerical investigation. In [3,6], the authors applied finite difference schemes to the Dirichlet problem; for an optimal error analysis of finite difference and finite element schemes in the case of Neumann- or a Robin-type bottom boundary condition $c f$. [4,8]. More recently, space-time discontinuous in time Galerkin methods for the Dirichlet problem in $\mathbb{R}^{n}$ have been analyzed in [7]; cf. also in [6], for finite element methods of optimal accuracy 
in the multi-dimensional case with Dirichlet conditions. In [1], the Neumann boundary condition was modified in a Robin-type one to ensure well-posedness of the (PE) problem posed on a rigid bottom; this model extended in two dimensions has been analyzed in [9], where by proving a global elliptic regularity result the authors derived optimal error estimates for the proposed finite element scheme.

Without restrictions on the bottom profile $s$, the well posedness of the (PE) with a Neumann condition (as an initial and boundary value problem) remains open, while as we shall see in the sequel, there appear certain technical obstacles even when we treat the numerical scheme.

\subsection{The transformed problem}

In order to derive an equivalent problem posed on a rectangle as in $[4,6,7,9,17]$, we apply the horizontal bottom transformation

$$
z:=\frac{x}{s(r, \vartheta)}
$$

Furthermore, we define

$$
q(r, \vartheta):=-\frac{\ln s}{2}, \quad \Psi(r, x, \vartheta)=e^{q(r, \vartheta)} U(r, z, \vartheta) .
$$

After this change of variables the ibvp (1.1)-(1.4) takes the equivalent formulation for $\mathcal{D}:=[0,1] \times\left[\vartheta_{1}, \vartheta_{2}\right]$,

$$
\begin{aligned}
& U_{r}=\mathbf{A} U_{z z}+\mathbf{B} U_{z \vartheta}+\mathbf{C} U_{\vartheta \vartheta}+\mathbf{D} U_{z}+\mathbf{E} U_{\vartheta}+\mathrm{i} \beta U+f \text { in }\left[R_{0}, R\right] \times \mathcal{D} \\
& U=0 \text { at } z=0, \quad \text { and at } \vartheta=\vartheta_{1}, \vartheta_{2}, \\
& {\left[\mathbf{A} U_{z}+\frac{\mathbf{B}}{2} U_{\vartheta}\right]+a_{1} U_{z}+a_{2} U+a_{3} U_{r}=0 \text { at } z=1,} \\
& U=U_{0}(z, \vartheta) \quad \text { at } \quad r=R_{0},
\end{aligned}
$$

where $a_{1}, a_{2}, a_{3}$ are functions of $r, \vartheta$, and $\mathbf{A}=\mathbf{A}(r, z, \vartheta), \mathbf{B}=\mathbf{B}(r, z, \vartheta), \mathbf{C}=\mathbf{C}(r), \mathbf{D}=\mathbf{D}(r, z, \vartheta), \mathbf{E}=\mathbf{E}(r, \vartheta)$, $f=f(r, z, \vartheta)$. Also, $c f$. [5], for $\tilde{a} \neq 0$, then $\mathbf{A}, \mathbf{B}, \mathbf{C}, \mathbf{D}, \mathbf{E}, \beta, a_{1}, a_{2}, a_{3}$, are given by

$$
\begin{aligned}
& \mathbf{A}(z, r, \vartheta):=\mathrm{i} \tilde{a}\left\{\frac{1}{s^{2}}+\frac{z^{2} s_{\vartheta}^{2}}{r^{2} s^{2}}\right\}, \quad \mathbf{B}(z, r, \vartheta):=-\frac{2 \mathrm{i} \tilde{a}}{r^{2}} z \frac{s_{\vartheta}}{s}, \mathbf{C}(r):=\frac{\mathrm{i} \tilde{a}}{r^{2}}, \\
& \mathbf{D}(z, r, \vartheta):=z \frac{s_{r}}{s}-\frac{\mathrm{i} \tilde{a}}{r^{2}} \frac{z}{s^{2}}\left(s_{\vartheta \vartheta} s-2 s_{\vartheta}^{2}\right)-2 \frac{\mathrm{i} \tilde{a}}{r^{2}} z \frac{s_{\vartheta}}{s} q_{\vartheta}, \quad \mathbf{E}(r, \vartheta):=2 q_{\vartheta} \frac{\mathrm{i} \tilde{a}}{r^{2}} \\
& \beta:=\beta_{R}+\mathrm{i} \beta_{I}, \\
& a_{1}(r, \vartheta)=\frac{\mathrm{i} \tilde{a} s_{r}^{2}}{s^{2}}, \quad a_{2}(r, \vartheta)=\frac{\mathrm{i} \tilde{a}}{s} \gamma \text { for } \gamma(r, \vartheta):=\frac{s_{r}^{2}}{2 s}+\frac{s_{\vartheta}^{2}}{2 r^{2} s}, \quad a_{3}(r, \vartheta)=-s_{r} \frac{\mathrm{i} \tilde{a}}{s} .
\end{aligned}
$$

Remark 1.1. The first change of variables, (1.6), transforms the noncylindrical domain into a cylindrical one fact that somehow simplifies the application of numerical schemes, since range and space discretizations can be independent; remind that range is a time-like variable. Of course, the resulting equation becomes more complicated.

The second exponential transformation (1.7) together with the specific definition of $q$ has been introduced in [17]. From a mathematical point of view, it is related to the variable bottom boundary condition, which is for this problem a Neumann one. It provides a simpler weak formulation for the continuous problem, while it is crucial for the stability of the constructed numerical scheme (see also the Rems. 2.1, and 3.4 in Sects. 2 and 3 respectively).

Remark 1.2. Note that $f=0$ for the specific transformed problem but for generalization purposes we shall define $f$ as an arbitrary complex function. In order to treat the (PE) problem (1.1) in a more general setting, we introduce the complex function $\beta_{\Psi}:=\operatorname{Re} \beta_{\Psi}+\mathrm{i} \operatorname{Im} \beta_{\Psi}$ as the coefficient of the zero order term; usually 
the imaginary part of $\beta_{\Psi}$ refers to the absorption of the sound signal in the ocean (attenuation), [15], while $\frac{k_{0}}{2}\left(n_{\mathrm{ref}}^{2}-1\right)$ may define $\operatorname{Re} \beta_{\Psi}$.

The formulae of $\tilde{a}, \beta$ and $U_{0}$ will not be used in this paper, we present them though for completeness; these are given as follows:

$$
\begin{aligned}
& \tilde{a}:=\frac{1}{2 k_{0}}, \\
& \beta_{R}:=\operatorname{Re} \beta_{\Psi}+\frac{\tilde{a}}{r^{2}} \operatorname{Re}\left(q_{\vartheta}^{2}+q_{\vartheta \vartheta}\right)-\operatorname{Im} q_{r}, \\
& \beta_{I}:=\operatorname{Im} \beta_{\Psi}+\frac{\tilde{a}}{r^{2}} \operatorname{Im}\left(q_{\vartheta}^{2}+q_{\vartheta \vartheta}\right)+\operatorname{Re} q_{r} \\
& U_{0}(z, \vartheta):=e^{-q\left(R_{0}, \vartheta\right)} \Psi_{0}\left(z s\left(R_{0}, \vartheta\right), \vartheta\right) .
\end{aligned}
$$

\subsection{Main results}

The problem analyzed although being linear is very difficult due to the Neumann boundary condition posed on a noncylindrical domain. More specifically in this condition appears a dynamical term together with first order terms in space variables and the same holds after the horizontal bottom transformation in

$$
\left[\mathbf{A} U_{z}+\frac{\mathbf{B}}{2} U_{\vartheta}\right]+a_{1} U_{z}+a_{2} U+a_{3} U_{r}=0 \quad \text { at } \quad z=1 .
$$

When the problem is written in weak formulation, this leads to trace integrals of space derivatives that are of lower regularity and thus hardly estimated; in fact only those coming from $\left[\mathbf{A} U_{z}+\frac{\mathbf{B}}{2} U_{\vartheta}\right]$ are eliminated. In addition, the resulting p.d.e. has complex coefficients depending on all variables.

We formulate implicit finite element schemes for the ibvp (1.8). The treatment of the dynamical boundary condition needs very refined estimates that are proven under the assumption of higher regularity for the finite element space. More specifically, we use a cubic splines approximation in space variables; this was not the case for the one-dimensional problem analyzed in [8], where a piece-wise linear approximation gave a second order optimal error in the $L^{2}$ norm. Furthermore, we apply a Crank-Nicolson discretization for the time-like variable $r$ and estimate the semidiscrete and fully discrete error in the $H^{1}$ norm.

For a general upsloping bottom topography, where $s$ is a function of the range $r$ and azimuth $\vartheta$ and satisfies (1.5), we prove an $H^{1}$ error of order $\mathcal{O}\left(h^{3-\frac{1}{2}}+k^{2}\right)$, for $h$ and $k$ the discretization parameters in space and range respectively. Thus even though the scheme loses $\frac{1}{2}$ from being optimal, it is a high order scheme in space, while for $h=\mathcal{O}(k)$ the error in the $L^{2}$ norm is of second order of accuracy. In other words, the dynamical Neumann condition penalizes only slightly the order of accuracy.

In addition, if $s$ depends only on azimuth we derive an optimal $H^{1}$ error of order $\mathcal{O}\left(h^{3}+k^{2}\right)$.

The rest of this paper is as follows: In Section 2 we express the ibvp (1.8) in a weak formulation for which we prove uniqueness of solution. Here, we define an $H^{1}$-type hermitian sesquilinear form. Further, we analyze the form's properties, mainly in regard to the higher regularity of the input data, to observe that more regular inputs $\left(H^{2}\right)$ lead to better estimates. The next Section, 3, contains the analysis of a semidiscrete scheme in space variables, and presents the detailed proof of $H^{1}$-estimates of the error. Finally, in Section 4 we apply a Crank-Nicolson method in range. By deriving an $H^{1}$-stability result, we prove uniqueness of solution and estimate the fully discrete error.

\section{WEAK FORMULATION}

Let $(\cdot, \cdot)$ denote the usual $L^{2}(\mathcal{D})$ inner product, and $\|\cdot\|,\|\cdot\|_{m}, m \in \mathbb{N}^{*}$ the norms in $L^{2}(\mathcal{D})$ and $H^{m}(\mathcal{D})$ respectively.

For $r$ fixed, we define

$$
\widetilde{H}_{0}^{1}(\mathcal{D}):=\left\{u \in H^{1}(\mathcal{D}): u=0 \text { at } z=0, \text { and at } \vartheta=\vartheta_{1}, \vartheta_{2}\right\}
$$


where $H^{1}(\mathcal{D})$ is the usual complex Sobolev space. Multiplying the p.d.e of (1.8) with $\bar{\phi} \in \widetilde{H}_{0}^{1}(\mathcal{D})$, integrating and using the boundary conditions we get the following weak formulation:

$$
\begin{aligned}
\left(U_{r}, \phi\right)= & -\left(\mathbf{A} U_{z}, \phi_{z}\right)-\left(\mathbf{C} U_{\vartheta}, \phi_{\vartheta}\right)-\frac{1}{2}\left\{\left(\mathbf{B} U_{z}, \phi_{\vartheta}\right)+\left(\mathbf{B} U_{\vartheta}, \phi_{z}\right)\right\} \\
& +\left(\left\{\mathbf{D}-\mathbf{A}_{z}-\frac{\mathbf{B}_{\vartheta}}{2}\right\} U_{z}, \phi\right)+\mathrm{i}(\beta U, \phi)+(f, \phi)+\int_{z=1}\left[\mathbf{A} U_{z}+\frac{\mathbf{B}}{2} U_{\vartheta}\right] \bar{\phi} \mathrm{d} \vartheta .
\end{aligned}
$$

Note that for $z=1$ we have

$$
\mathbf{A} U_{z}+\frac{\mathbf{B}}{2} U_{\vartheta}=\mathrm{i} \tilde{a} \frac{s_{r}}{s}\left(U_{r}-\frac{s_{r}}{s} U_{z}\right)-\frac{\mathrm{i} \tilde{a}}{s} \gamma(r, \vartheta) U
$$

Thus, since

$$
\mathbf{D}-\mathbf{A}_{z}-\frac{\mathbf{B}_{\vartheta}}{2}=z \frac{s_{r}}{s},
$$

the weak formulation becomes

$$
\begin{aligned}
\left(U_{r}-z \frac{s_{r}}{s} U_{z}-\mathrm{i} \beta U, \phi\right)= & -\left(\mathbf{A} U_{z}, \phi_{z}\right)-\left(\mathbf{C} U_{\vartheta}, \phi_{\vartheta}\right)-\frac{1}{2}\left\{\left(\mathbf{B} U_{z}, \phi_{\vartheta}\right)+\left(\mathbf{B} U_{\vartheta}, \phi_{z}\right)\right\} \\
& +\mathrm{i} \tilde{a} \int_{z=1}\left[\frac{s_{r}}{s}\left(U_{r}-\frac{s_{r}}{s} U_{z}\right)-\frac{1}{s} \gamma(r, \vartheta) U\right] \bar{\phi} \mathrm{d} \vartheta+(f, \phi),
\end{aligned}
$$

for any $\phi \in \widetilde{H}_{0}^{1}(\mathcal{D})$.

We shall prove that $(2.3)$ admits at most one solution in $\widetilde{H}_{0}^{1}(\mathcal{D}) \cap H^{2}(\mathcal{D})$, under the assumptions (1.5) for $s$.

Remark 2.1. Note that the right-hand side of (2.2) admits this formula, which coincides with a specific term of the Neumann condition at $z=1$, due to the exponential transformation (1.7). Furthermore, the presence of $z$ is important since then $U_{r}-z \frac{s_{r}}{s} U_{z}-\mathrm{i} \beta U \in \widetilde{H}_{0}^{1}(\mathcal{D})$ and can be used as a test function in the weak formulation when proving uniqueness in Theorem 2.6; this will result in an imaginary trace integral for the first order terms.

For any $r$ in $\left[R_{0}, R\right]$, let $\mathcal{B}(r ; v, w):\left[R_{0}, R\right] \times H^{1}(\mathcal{D}) \times H^{1}(\mathcal{D}) \rightarrow \mathbb{C}$ be the following sesquilinear form, for $s$, $s_{\vartheta}$ defined on $r$ :

$$
\mathcal{B}(r ; v, w):=\left(\frac{1}{s^{2}}\left(1+\frac{s_{\vartheta}^{2}}{r^{2}} z^{2}\right) v_{z}, w_{z}\right)+\frac{1}{r^{2}}\left(v_{\vartheta}, w_{\vartheta}\right)-\frac{1}{r^{2}}\left\{\left(z \frac{s_{\vartheta}}{s} v_{z}, w_{\vartheta}\right)+\left(z \frac{s_{\vartheta}}{s} v_{\vartheta}, w_{z}\right)\right\} .
$$

Since all the appearing functions of $z, r, \vartheta$ are real, it follows that $\mathcal{B}$ is hermitian, i.e.

$$
\mathcal{B}(r ; v, w)=\overline{\mathcal{B}(r ; w, v)} .
$$

Using the definition of $\mathcal{B}$, the weak problem (2.3) is equivalently written as

$$
\begin{aligned}
\left(U_{r}-z \frac{s_{r}}{s} U_{z}-\mathrm{i} \beta U, \phi\right)= & -\mathrm{i} \tilde{a} \mathcal{B}(r ; U, \phi) \\
& +\mathrm{i} \tilde{a} \int_{z=1}\left[\frac{s_{r}}{s}\left(U_{r}-\frac{s_{r}}{s} U_{z}\right)-\frac{1}{s} \gamma(r, \vartheta) U\right] \bar{\phi} \mathrm{d} \vartheta+(f, \phi),
\end{aligned}
$$

for any $\phi \in \widetilde{H}_{0}^{1}(\mathcal{D})$ with $U \in \widetilde{H}_{0}^{1}(\mathcal{D}) \cap H^{2}(\mathcal{D})$ and $U=U_{0}(z, \vartheta)$ at $r=R_{0}$.

Throughout the rest of this paper the letter $C$ will denote generic constants independent of the discretization parameters.

Due to the definitions of $\mathbf{A}, \mathbf{B}$, and $\mathbf{C}$, the stationary problem is elliptic and the sesquilinear form is coercive. Furthermore, we obtain the next important lemma proven analytically in $[5,9]$. 
Lemma 2.2. If $v \in H^{1}(\mathcal{D})$, then there exist constants $C_{1}, C_{2}>0$ such that

$$
C_{1}\|v\|_{1}^{2} \leq \mathcal{B}(r ; v, v) \leq C_{2}\|v\|_{1}^{2} \text {, for any } r \in\left[R_{0}, R\right] .
$$

In addition, by straightforward calculations the next lemma follows, $c f .[5,9]$.

Lemma 2.3. If $v, v_{r} \in H^{1}(\mathcal{D})$ then there exists a constant $C_{0}>0$ such that

$$
\frac{1}{2} \partial_{r} \mathcal{B}(r ; v, v) \leq \operatorname{Re} \mathcal{B}\left(r ; v, v_{r}\right)+C_{0}\|v\|_{1}^{2} .
$$

We remind that if $\Omega$ is a bounded domain of Lipschitz boundary and $1 \leq p \leq \infty$ then

$$
\|v\|_{L^{p}(\partial \Omega)} \leq C\|v\|_{L^{p}(\Omega)}^{1-1 / p}\|v\|_{W_{p}^{1}(\Omega)}^{1 / p},
$$

for any $v \in W_{p}^{1}(\Omega), c f$. [10]. So, for $p=2$ and since $W_{2}^{1}(\mathcal{D}):=H^{1}(\mathcal{D})$ we have

$$
\int_{z=1}|v|^{2} \mathrm{~d} \vartheta \leq C\|v\|\|v\|_{1}
$$

for any $v \in H^{1}(\mathcal{D})$. This inequality will be frequently used when we estimate the appearing trace integral terms.

Assuming higher regularity for the variables of the form $\mathcal{B}$ we prove the next lemma. In view of relation (2.8), $H^{2}(\mathcal{D})$ regularity is sufficient.

Lemma 2.4. If $\phi \in \widetilde{H}_{0}^{1}(\mathcal{D}) \cap H^{2}(\mathcal{D})$ then there exists a constant $C>0$ such that

$$
\begin{aligned}
\operatorname{Re} \mathcal{B}\left(r ; \phi, z \frac{s_{r}}{s} \phi_{z}\right) \leq & C\|\phi\|_{1}^{2}+\frac{1}{2} \int_{z=1} \frac{s_{r}}{s^{3}}\left\{1+\frac{s_{\vartheta}^{2}}{r^{2}}\right\}\left|\phi_{z}\right|^{2} \mathrm{~d} \vartheta \\
& +\frac{1}{2} \frac{1}{r^{2}} \int_{z=1} \frac{s_{r}}{s}\left|\phi_{\vartheta}\right|^{2} \mathrm{~d} \vartheta-\frac{1}{r^{2}} \operatorname{Re} \int_{z=1} \frac{s_{\vartheta} s_{r}}{s^{2}} \phi_{z} \bar{\phi}_{\vartheta} \mathrm{d} \vartheta .
\end{aligned}
$$

Proof. The function $z \frac{s_{r}}{s} \phi_{z} \in \widetilde{H}_{0}^{1}(\mathcal{D})$ since it is zero at $z=0, \vartheta=\vartheta_{1}, \vartheta=\vartheta_{2}$; hence, the quantity $\mathcal{B}\left(r ; \phi, z \frac{s_{r}}{s} \phi_{z}\right)$ is well defined. We use the definition of $\mathcal{B}$, and take real parts to obtain

$$
\begin{aligned}
\operatorname{Re} \mathcal{B}\left(r ; \phi, z \frac{s_{r}}{s} \phi_{z}\right) \leq & C\|\phi\|_{1}^{2}+\operatorname{Re}\left(\frac{1}{s^{2}}\left\{1+z^{2} \frac{s_{\vartheta}^{2}}{r^{2}}\right\} \phi_{z}, z \frac{s_{r}}{s} \phi_{z z}\right)+\frac{1}{r^{2}} \operatorname{Re}\left(\phi_{\vartheta}, z \frac{s_{r}}{s} \phi_{z \vartheta}\right) \\
& -\frac{1}{r^{2}} \operatorname{Re}\left\{\left(z \frac{s_{\vartheta}}{s} \phi_{z}, z \frac{s_{r}}{s} \phi_{z \vartheta}\right)+\left(z \frac{s_{\vartheta}}{s} \phi_{\vartheta}, z \frac{s_{r}}{s} \phi_{z z}\right)\right\} .
\end{aligned}
$$

Further, we compute every real part at the right-hand side of the above inequality by applying integration by parts to each of these terms and using the boundary conditions, to get the result.

Remark 2.5. It holds that if $a, b, c$ are real and $|a||b|>\frac{|c|^{2}}{4}$ then

$$
|a||x|^{2}+|b||y|^{2}+c \operatorname{Re}\{x \bar{y}\} \geq|a||x|^{2}+|b||y|^{2}-|c||x||y| \geq 0,
$$

for any complex $x, y$. Hence, since

$$
\frac{1}{2} \frac{1}{s^{3}}\left\{1+\frac{s_{\vartheta}^{2}}{r^{2}}\right\} \frac{1}{2} \frac{1}{r^{2}} \frac{1}{s}>\frac{1}{4} \frac{1}{r^{4}} \frac{s_{\vartheta}^{2}}{s^{2} s^{2}},
$$

then under the assumptions (1.5) for $s$ (upsloping bottom), Lemma 2.4 gives

$$
\operatorname{Re} \mathcal{B}\left(r ; \phi, z \frac{s_{r}}{s} \phi_{z}\right) \leq C\|\phi\|_{1}^{2},
$$

for any $\phi \in \widetilde{H}_{0}^{1}(\mathcal{D}) \cap H^{2}(\mathcal{D})$. 
Note that without (1.5) the estimate would only be

$$
\operatorname{Re} \mathcal{B}\left(r ; \phi, z \frac{s_{r}}{s} \phi_{z}\right) \leq C\|\phi\|_{1}\|\phi\|_{2} .
$$

The estimate (2.9) which is true for the upsloping case is used to establish uniqueness of weak solution. In addition, it motivates in the sequel the choice of higher regularity $\left(H^{2}\right)$ for the finite element space and permits us to estimate the numerical error.

For the sake of a simpler notation and for the rest of this paper, the symbol $\mathcal{B}(v, w)$, whenever is used, will denote $\mathcal{B}(r ; v, w)$.

The next theorem establishes uniqueness of solution for the weak formulation (2.5) in the upsloping bottom case.

Theorem 2.6. Under the assumptions (1.5) for $s$ (upsloping bottom) the problem (2.5) admits at most one solution $U$ in $\widetilde{H}_{0}^{1}(\mathcal{D}) \cap H^{2}(\mathcal{D})$.

Proof. Obviously, since the non-homogeneous problem with general $f$ is linear, it is sufficient to prove uniqueness for $f=0$.

In $(2.5)$ we set $f:=0$ and define

$$
\phi:=U_{r}-z \frac{s_{r}}{s} U_{z}-\mathrm{i} \beta U \in \widetilde{H}_{0}^{1}(\mathcal{D}) .
$$

Furthermore, we take imaginary parts and obtain

$$
\begin{aligned}
\operatorname{Im}\left\{\tilde{a}^{-1}\|\phi\|^{2}\right\}=0= & -\operatorname{Re} \mathcal{B}\left(U, U_{r}\right)+\operatorname{Re} \mathcal{B}\left(U, z \frac{s_{r}}{s} U_{z}\right)+\operatorname{Re} \mathcal{B}(U, \mathrm{i} \beta U) \\
& +\int_{z=1} \frac{s_{r}}{s}|\phi+\mathrm{i} \beta U|^{2} \mathrm{~d} \vartheta+\operatorname{Re} \int_{z=1} \frac{s_{r}}{s}[\phi+\mathrm{i} \beta U][\overline{-\mathrm{i} \beta U}] \mathrm{d} \vartheta \\
& -\operatorname{Re} \int_{z=1} \frac{1}{s} \gamma(r, \vartheta) U[\overline{\phi+\mathrm{i} \beta U}] \mathrm{d} \vartheta-\operatorname{Re} \int_{z=1} \frac{1}{s} \gamma(r, \vartheta) U[\overline{-\mathrm{i} \beta U}] \mathrm{d} \vartheta .
\end{aligned}
$$

Therefore,

$$
\begin{aligned}
\operatorname{Re} \mathcal{B}\left(U, U_{r}\right)= & \operatorname{Re} \mathcal{B}\left(U, z \frac{s_{r}}{s} U_{z}\right)+\operatorname{Re} \mathcal{B}(U, \mathrm{i} \beta U) \\
& +\int_{z=1} \frac{s_{r}}{s}|\phi+\mathrm{i} \beta U|^{2} \mathrm{~d} \vartheta+\operatorname{Re} \int_{z=1} \frac{s_{r}}{s}[\phi+\mathrm{i} \beta U][\overline{-\mathrm{i} \beta U}] \mathrm{d} \vartheta \\
& -\operatorname{Re} \int_{z=1} \frac{1}{s} \gamma(r, \vartheta) U[\overline{\phi+\mathrm{i} \beta U}] \mathrm{d} \vartheta-\operatorname{Re} \int_{z=1} \frac{1}{s} \gamma(r, \vartheta) U[\overline{-\mathrm{i} \beta U}] \mathrm{d} \vartheta \\
\leq & C\|U\|_{1}^{2}+\operatorname{Re} \mathcal{B}(U, \mathrm{i} \beta U)+C\|U\|\|U\|_{1},
\end{aligned}
$$

where we used Remark 2.5 since $U \in \widetilde{H}_{0}^{1}(\mathcal{D}) \cap H^{2}(\mathcal{D})$, together with the trace inequality (2.8) and the upsloping bottom condition (1.5). Here, the non-positive trace term is

$$
\int_{z=1} \frac{s_{r}}{s}|\phi+\mathrm{i} \beta U|^{2} \mathrm{~d} \vartheta
$$

and is used to bound all the other trace integrals. Note that even when $s_{r}=0$ and this term vanishes the same happens for all traces also since by (1.5) $s_{\vartheta}=0$ and thus $\gamma=0$; this is the reason for assuming that when $s_{r}(r, \vartheta)$ is zero for some $r, \vartheta$ then $s_{\vartheta}(r, \vartheta)$ is zero also. 
We use now Lemma 2.3 for $v:=U \in H^{1}(\mathcal{D})$ and obtain

$$
\frac{1}{2} \partial_{r} \mathcal{B}(U, U)-C_{0}\|U\|_{1}^{2} \leq \operatorname{Re} \mathcal{B}\left(U, U_{r}\right)
$$

Hence, the above relation combined with (2.10) gives

$$
\frac{1}{2} \partial_{r} \mathcal{B}(U, U)-C_{0}\|U\|_{1}^{2} \leq \operatorname{Re} \mathcal{B}\left(U, U_{r}\right) \leq C\|U\|_{1}^{2}+\operatorname{Re} \mathcal{B}(U, \mathrm{i} \beta U)+C\|U\|\|U\|_{1},
$$

for some $C_{0}>0$.

Evidently, since $U \in H^{1}(\mathcal{D})$ then by $(2.4),(2.11)$ and Lemma 2.2 we obtain

$$
\frac{1}{2} \partial_{r} \mathcal{B}(U, U) \leq C \mathcal{B}(U, U) .
$$

We integrate (2.12) and get

$$
\mathcal{B}(U, U) \leq C \mathcal{B}\left(U_{0}, U_{0}\right) .
$$

Applying again Lemma 2.2 in both sides, we have

$$
\|U\|_{1} \leq C\left\|U_{0}\right\|_{1},
$$

and so,

$$
\|U\| \leq C\left\|U_{0}\right\|_{1}
$$

i.e. uniqueness of weak solution in $\widetilde{H}_{0}^{1}(\mathcal{D}) \cap H^{2}(\mathcal{D})$.

Remark 2.7. Usually in evolutionary equations, cf. [14] for parabolic initial and boundary value problems, a weak solution and its evolutionary derivative, if they exist, are considered in evolutionary spaces. In the same spirit, for the problem presented here, a weak solution $U$ as a function of $r, z, \vartheta$, if exists, should be in $L^{2}\left(R_{0}, R ; \mathcal{H}\right)$ for $\mathcal{H}:=\widetilde{H}_{0}^{1}(\mathcal{D}) \cap H^{2}(\mathcal{D})$ and $U_{r}$ in $L^{2}\left(R_{0}, R ; \mathcal{H}^{-1}\right)$ where $\mathcal{H}^{-1}$ is the dual space of $\mathcal{H}$.

When proving uniqueness of weak solution, we considered something stronger for $U$ : for any $r \in\left(R_{0}, R\right)$ fixed, $U(r, \cdot)$ in the Sobolev space $\mathcal{H}$ which gives that $U \in L^{2}\left(R_{0}, R ; \mathcal{H}\right)$ since the range interval is bounded.

Of course, we only treated the uniqueness question. A proof of existence of weak solution for the continuous problem, is not in the aims of this paper. When the bottom is only a function of range the problem is wellposed, [1,2]. We refer also to the existing bibliography for the Heat equation with dynamical boundary conditions conditions, [13,19], and to the book of Evans [14].

\section{SemidiscretizATion in DEPTH AND AZIMUth}

Let $S_{h}$ be a finite dimensional subspace of $\widetilde{H}_{0}^{1}(\mathcal{D}) \cap H^{2}(\mathcal{D})$ consisting of complex-valued functions that are polynomials of degree at most $\tau-1 \geq 3$ (for example cubic splines for $\tau=4$ ) in each element of a quasi-uniform partition of $\mathcal{D}$ with maximum diameter $h \in\left(0, h_{\star}\right]$. Then, [10], the following approximation property holds for $s=1, \ldots, \tau, \quad \forall v \in H^{s}(\mathcal{D}), \quad \forall h \in\left(0, h_{\star}\right]:$

$$
\inf _{\chi \in S_{h}}\left\{\|v-\chi\|+h\|v-\chi\|_{1}\right\} \leq C h^{s}\|v\|_{s} .
$$

Also, we assume that the following inverse inequality holds

$$
\|\chi\|_{1} \leq C h^{-1}\|\chi\| \quad \forall \chi \in S_{h}, \quad \forall h \in\left(0, h_{\star}\right],
$$

which is true since the partition of $\mathcal{D}$ is quasi-uniform [10]. 
We seek $U_{h}:\left[R_{0}, R\right] \rightarrow S_{h}$ such that

$$
\begin{aligned}
& \left(\partial_{r} U_{h}-R_{h}\left(z \frac{s_{r}}{s} U_{h z}+\mathrm{i} \beta U_{h}\right), \phi\right)=-\mathrm{i} \tilde{a} \mathcal{B}\left(U_{h}, \phi\right) \\
& +\mathrm{i} \tilde{a} \int_{z=1}\left[\frac{s_{r}}{s} R_{h}\left(\partial_{r} U_{h}-z \frac{s_{r}}{s} U_{h z}\right)-\frac{1}{s} \gamma(r, \vartheta) U_{h}\right] \bar{\phi} \mathrm{d} \vartheta+(f, \phi),
\end{aligned}
$$

for any $\phi \in S_{h}, R_{0}<r \leq R$, with $U_{h}\left(R_{0}\right)$ a suitable approximation of $U_{0}$ in $S_{h}$. Here, $R_{h}: \widetilde{H}_{0}^{1}(\mathcal{D}) \rightarrow S_{h}$ is defined to satisfy for any $v \in \widetilde{H}_{0}^{1}(\mathcal{D})$

$$
\mathcal{B}\left(R_{h} v, \phi\right)=\mathcal{B}(v, \phi) \text { for any } \phi \in S_{h} .
$$

Remark 3.1. The proposed semidiscrete scheme is implicit because it uses the projection induced by $\mathcal{B}$. As we shall see in the sequel, it mimics well the weak formulation of the continuous problem where when proving $H^{1}$ stability of solution we used $U_{r}-z \frac{s_{r}}{s} U_{z}+\mathrm{i} \beta U$ as test function. Here, the analogous element in $S_{h}$ is $\partial_{r} U_{h}-R_{h}\left(z \frac{s_{r}}{s} U_{h z}+\mathrm{i} \beta U_{h}\right) \in H^{2}(\mathcal{D})$.

Remark 3.2. Observe that $|\mathcal{B}(v, w)| \leq C\|v\|_{1}\|w\|_{1}$ for any $v, w \in \widetilde{H}_{0}^{1}(\mathcal{D})$. Lemma 2.2, since $v \in \widetilde{H}_{0}^{1}(\mathcal{D}) \subset$ $H^{1}(\mathcal{D})$, gives that $\mathcal{B}(v, v) \geq C\|v\|_{1}^{2}$. Hence, by Lax-Milgram Lemma the projection operator $R_{h}$ is well defined.

The next result establishes the existence of a unique solution for the scheme (3.3).

Proposition 3.3. Under the assumptions (1.5) (upsloping bottom), there exists a unique solution $U_{h} \in S_{h}$ for the semidiscrete scheme (3.3), which satisfies if $f=0$

$$
\left\|U_{h}\right\|_{1} \leq C\left\|U_{h}\left(R_{0}\right)\right\|_{1} .
$$

Proof. Since the problem is linear, in order to prove uniqueness it is sufficient to consider $f=0$. So, in (3.3), we set $f=0$ and

$$
\phi:=\partial_{r} U_{h}-R_{h}\left(z \frac{s_{r}}{s} U_{h z}+\mathrm{i} \beta U_{h}\right) ;
$$

$\phi \in S_{h}$ because $z \frac{s_{r}}{s} U_{h z} \in \widetilde{H}_{0}^{1}(\mathcal{D})$ and $U_{h} \in S_{h} \subset \widetilde{H}_{0}^{1}(\mathcal{D}) \cap H^{2}(\mathcal{D})$. So, we have

$$
\begin{aligned}
\|\phi\|^{2}= & \left\|\partial_{r} U_{h}-R_{h}\left(z \frac{s_{r}}{s} U_{h z}+\mathrm{i} \beta U_{h}\right)\right\|^{2} \\
= & -\mathrm{i} \tilde{a} \mathcal{B}\left(U_{h}, \partial_{r} U_{h}\right)+\mathrm{i} \tilde{a} \mathcal{B}\left(U_{h}, R_{h}\left(z \frac{s_{r}}{s} U_{h z}\right)\right)+\mathrm{i} \tilde{a} \mathcal{B}\left(U_{h}, R_{h}\left(\mathrm{i} \beta U_{h}\right)\right) \\
& +\mathrm{i} \tilde{a} \int_{z=1}\left\{\frac{s_{r}}{s}\left[\partial_{r} U_{h}-R_{h}\left(z \frac{s_{r}}{s} U_{h z}\right)\right]-\frac{1}{s} \gamma(r, \vartheta) U_{h}\right\}\left[\overline{\partial_{r} U_{h}-R_{h}\left(z \frac{s_{r}}{s} U_{h z}+\mathrm{i} \beta U_{h}\right)}\right] \mathrm{d} \vartheta .
\end{aligned}
$$

But by the projection operator definition, it holds that

$$
\mathcal{B}\left(U_{h}, R_{h}\left(z \frac{s_{r}}{s} U_{h z}\right)\right)=\overline{\mathcal{B}\left(R_{h}\left(z \frac{s_{r}}{s} U_{h z}\right), U_{h}\right)}=\overline{\mathcal{B}\left(z \frac{s_{r}}{s} U_{h z}, U_{h}\right)}=\mathcal{B}\left(U_{h}, z \frac{s_{r}}{s} U_{h z}\right),
$$

while

$$
\mathcal{B}\left(U_{h}, R_{h}\left(\mathrm{i} \beta U_{h}\right)\right)=\overline{\mathcal{B}\left(R_{h}\left(\mathrm{i} \beta U_{h}\right), U_{h}\right)}=\overline{\mathcal{B}\left(\mathrm{i} \beta U_{h}, U_{h}\right)}=\mathcal{B}\left(U_{h}, \mathrm{i} \beta U_{h}\right) .
$$

Replacing (3.6) and (3.7) in (3.5), and using

$$
\partial_{r} U_{h}-R_{h}\left(z \frac{s_{r}}{s} U_{h z}\right)=\phi+R_{h}\left(\mathrm{i} \beta U_{h}\right),
$$


at the right-hand side, we have

$$
\begin{aligned}
\|\phi\|^{2}= & \left\|\partial_{r} U_{h}-R_{h}\left(z \frac{s_{r}}{s} U_{h z}+\mathrm{i} \beta U_{h}\right)\right\|^{2} \\
= & -\mathrm{i} \tilde{a} \mathcal{B}\left(U_{h}, \partial_{r} U_{h}\right)+\mathrm{i} \tilde{a} \mathcal{B}\left(U_{h}, z \frac{s_{r}}{s} U_{h z}\right)+\mathrm{i} \tilde{a} \mathcal{B}\left(U_{h}, \mathrm{i} \beta U_{h}\right) \\
& \left.+\mathrm{i} \tilde{a} \int_{z=1}\left\{\frac{s_{r}}{s}\left[\partial_{r} U_{h}-R_{h}\left(z \frac{s_{r}}{s} U_{h z}\right)\right]-\frac{1}{s} \gamma(r, \vartheta) U_{h}\right\} \overline{\partial_{r} U_{h}-R_{h}\left(z \frac{s_{r}}{s} U_{h z}+\mathrm{i} \beta U_{h}\right)}\right] \mathrm{d} \vartheta \\
= & \mathrm{i} \tilde{a}\left[-\mathcal{B}\left(U_{h}, \partial_{r} U_{h}\right)+\mathcal{B}\left(U_{h}, z \frac{s_{r}}{s} U_{h z}\right)+\mathcal{B}\left(U_{h}, \mathrm{i} \beta U_{h}\right)\right] \\
& +\mathrm{i} \tilde{a} \int_{z=1}\left\{\frac{s_{r}}{s}\left[\phi+R_{h}\left(\mathrm{i} \beta U_{h}\right)\right]-\frac{1}{s} \gamma(r, \vartheta) U_{h}\right\} \bar{\phi} \mathrm{d} \vartheta .
\end{aligned}
$$

In the above, we take imaginary parts and use (1.5) to obtain

$$
\begin{aligned}
\operatorname{Re} \mathcal{B}\left(U_{h}, \partial_{r} U_{h}\right) \leq & \operatorname{Re} \mathcal{B}\left(U_{h}, z \frac{s_{r}}{s} U_{h z}\right)+\operatorname{Re} \mathcal{B}\left(U_{h}, \mathrm{i} \beta U_{h}\right) \\
& +C \int_{z=1}\left[\left|U_{h}\right|^{2}+\left|R_{h}\left(\mathrm{i} \beta U_{h}\right)\right|^{2}\right] \mathrm{d} \vartheta
\end{aligned}
$$

But for $v \in \widetilde{H}_{0}^{1}(\mathcal{D})$, it follows that

$$
C_{1}\left\|R_{h} v\right\|_{1}^{2} \leq C_{2}\left\|R_{h} v\right\|_{1}\|v\|_{1},
$$

for some positive constants $C_{1}, C_{2}$. So, we have

$$
\left\|R_{h} v\right\|_{1}^{2} \leq C\|v\|_{1} \text { for any } v \in \widetilde{H}_{0}^{1}(\mathcal{D}) .
$$

Using (3.10) in (3.9) and applying the trace inequality, we obtain

$$
\operatorname{Re} \mathcal{B}\left(U_{h}, \partial_{r} U_{h}\right) \leq \operatorname{Re} \mathcal{B}\left(U_{h}, z \frac{s_{r}}{s} U_{h z}\right)+C\left\|U_{h}\right\|_{1}^{2} .
$$

Relation (3.11) together with Lemma 2.3 for $U_{h} \in H^{1}(\mathcal{D})$ and Remark 2.5 (since $U_{h} \in \widetilde{H}_{0}^{1}(\mathcal{D}) \cap H^{2}(\mathcal{D})$ ), give for some $C_{0}>0$

$$
\begin{aligned}
\frac{1}{2} \frac{\mathrm{d}}{\mathrm{d} r} \mathcal{B}\left(U_{h}, U_{h}\right)-C_{0}\left\|U_{h}\right\|_{1}^{2} & \leq \operatorname{Re} \mathcal{B}\left(U_{h}, \partial_{r} U_{h}\right) \leq \operatorname{Re} \mathcal{B}\left(U_{h}, z \frac{s_{r}}{s} U_{h z}\right)+C\left\|U_{h}\right\|_{1}^{2} \\
& \leq C\left\|U_{h}\right\|_{1}^{2} .
\end{aligned}
$$

We integrate the above, use Lemma 2.2 and get for $f=0$

$$
\left\|U_{h}\right\|_{1} \leq C\left\|U_{h}\left(R_{0}\right)\right\|_{1} \text { (uniqueness, stability). }
$$

Finally, we shall prove that the semidiscrete scheme (3.3) for $f=0$, which is written in implicit form, is a linear first order ordinary differential system, where the matrix consisting of the coefficients of the derivatives is invertible. So, it has a solution which by stability is unique. As a result the non-homogeneous linear scheme has also a unique solution.

Indeed, for the homogeneous differential system we obtain

$$
\begin{aligned}
\left(\partial_{r} U_{h}, \phi\right)= & \left(R_{h}\left(\frac{s_{r}}{s} z U_{h z}-\mathrm{i} \beta U_{h}\right), \phi\right)-\mathrm{i} \tilde{a} \mathcal{B}\left(U_{h}, \phi\right) \\
& +\mathrm{i} \tilde{a} \int_{z=1}\left\{\frac{s_{r}}{s}\left[\partial_{r} U_{h}-R_{h}\left(z \frac{s_{r}}{s} U_{h z}\right)\right]-\frac{1}{s} \gamma(r, \vartheta) U_{h}\right\} \bar{\phi} \mathrm{d} \vartheta .
\end{aligned}
$$


Observe first that the projection operator $R_{h}$ is a linear operator taking values in a finite-dimensional space. Let $U_{h}=\sum_{i=1}^{N} c_{i}(r) \phi_{i}$, then (3.12) gives that

$$
B \overrightarrow{\dot{c}}(r)=C \vec{c}(r)
$$

with

Hence, $B$ has the form

$$
B_{i j}=\left(\phi_{i}, \phi_{j}\right)-\mathrm{i} \tilde{a} \int_{z=1} \frac{s_{r}}{s} \phi_{i} \bar{\phi}_{j} \mathrm{~d} \vartheta
$$

$$
B=A_{1}-\mathrm{i} \tilde{a} A_{2},
$$

where $\phi_{i}, \phi_{j} \in \mathbb{R}$, and $A_{1}$ is a real, symmetric and positive definite matrix, while $A_{2}$ is a real symmetric matrix. Therefore, $c f .[5,8]$, the matrix $B$ is invertible, and (3.12) admits a unique solution.

Remark 3.4. In order to prove stability we used the test function $\phi=\partial_{r} U_{h}-R_{h}\left(z \frac{s_{r}}{s} U_{h z}+\mathrm{i} \beta U_{h}\right) \in S_{h}$. This choice was possible and convenient for the same reasons explained in Remark 2.1, related to the specific exponential transformation (1.7). Uniqueness of weak solution for the initial continuous problem is in fact independent from (1.7), which just made the proof easier for the equivalent transformed one. The numerical scheme is of course defined after the implementation of (1.7), so its stability certainly depends on this transformation.

\subsection{Error estimates for the semidiscrete scheme}

Let $\varepsilon:=U_{h}-U$ be the error of the semidiscrete scheme, where $U_{h} \in S_{h}$ is the solution of (3.3) and $U$ is the solution of the weak problem (2.3).

For any $u \in H^{1}(\mathcal{D})$ we define

$$
\omega(u):=R_{h} u-u
$$

We write

$$
\varepsilon=\theta+\omega(U),
$$

with

$$
\theta:=U_{h}-R_{h} U \in S_{h}
$$

and $\omega(U):=R_{h} U-U$, where $R_{h}$ is the projection operator given by (3.4).

Considering the projection error $\omega$ and its derivatives in $r$, the next important lemma holds true; $c f .[5,9]$ for the analytical and very technical proof which is based on a global elliptic regularity result for complex elliptic problems with mixed Dirichlet and Robin conditions proven in [5,9].

Lemma 3.5. If $v \in \widetilde{H}_{0}^{1}(\mathcal{D}) \cap H^{s}(\mathcal{D})$ then for $\omega:=\omega(v)$ we have

$$
\begin{aligned}
& \|\omega\|_{1} \leq C h^{s-1}\|v\|_{s} \text { and }\|\omega\| \leq C h^{s}\|v\|_{s}, \\
& \left\|\omega_{r}\right\|_{1} \leq C h^{s-1}\left\{\|v\|_{s}+\left\|v_{r}\right\|_{s}\right\} \quad \text { and }\left\|\omega_{r}\right\| \leq C h^{s}\left\{\|v\|_{s}+\left\|v_{r}\right\|_{s}\right\}, \\
& \left\|\omega_{r r}\right\| \leq\left\|\omega_{r r}\right\|_{1} \leq C h^{s-1}\left\{\|v\|_{s}+\left\|v_{r}\right\|_{s}+\left\|v_{r r}\right\|_{s}\right\}
\end{aligned}
$$

for any $1 \leq s \leq \tau$, where $\omega=R_{h} v-v$ and $C>0$ is a constant independent of $v, h$.

Due to the fact that we seek an $H^{2}(\mathcal{D})$ semidiscrete solution we shall need estimates for the $H^{2}$ norm of $\omega$, presented at the following lemma.

Lemma 3.6. If $v \in \widetilde{H}_{0}^{1}(\mathcal{D}) \cap H^{s}(\mathcal{D}), 2 \leq s \leq 4$, then for $\omega:=\omega(v)$ we have

$$
\|\omega\|_{2} \leq C h^{s-2}\|v\|_{s} \text { and }\left\|\omega_{r}\right\|_{2} \leq C h^{s-2}\left\{\|v\|_{s}+\left\|v_{r}\right\|_{s}\right\}
$$

where $C>0$ is a constant independent of $v, h$. 
Proof. We assumed that $S_{h} \subseteq \widetilde{H}_{0}^{1}(\mathcal{D}) \cap H^{2}(D)$. Therefore, the interpolant $I_{h}: \widetilde{H}_{0}^{1}(\mathcal{D}) \cap H^{2}(\mathcal{D}) \rightarrow S_{h}$ satisfies

$$
\left\|I_{h} v-v\right\|+h\left\|I_{h} v-v\right\|_{1}+h^{2}\left\|I_{h} v-v\right\|_{2} \leq C h^{s}\|v\|_{s},
$$

for $2 \leq s \leq \tau$ and $v \in \widetilde{H}_{0}^{1}(\mathcal{D}) \cap H^{s}(\mathcal{D})$.

We have that $\tau=4$ (cubic splines [16]). Then for $v \in \widetilde{H}_{0}^{1}(\mathcal{D}) \cap H^{s}(\mathcal{D})$, using the inverse inequality in $S_{h}$ and the approximation property of the interpolant, together with Lemma 3.5, we obtain

$$
\begin{aligned}
\left\|R_{h} v-v\right\|_{2} \leq & \left\|R_{h} v-I_{h} v\right\|_{2}+\left\|I_{h} v-v\right\|_{2} \leq C h^{-2}\left\|R_{h} v-I_{h} v\right\|+c h^{s-2}\|v\|_{s} \\
& \leq C h^{-2}\left\|R_{h} v-v\right\|+c h^{-2}\left\|v-I_{h} v\right\|+C h^{s-2}\|v\|_{s} \\
& \leq C h^{-2} h^{s}\|v\|_{s}+c h^{-2} h^{s}\|v\|_{s}+C h^{s-2}\|v\|_{s} \leq C h^{s-2}\|v\|_{s},
\end{aligned}
$$

and

$$
\begin{aligned}
\left\|\left(R_{h} v-v\right)_{r}\right\|_{2} & =\left\|\left(R_{h} v\right)_{r}-v_{r}\right\|_{2} \leq\left\|\left(R_{h} v\right)_{r}-R_{h}\left(v_{r}\right)\right\|_{2}+\left\|R_{h}\left(v_{r}\right)-v_{r}\right\|_{2} \\
& \leq C h^{-2}\left\|\left(R_{h} v\right)_{r}-R_{h}\left(v_{r}\right)\right\|+C h^{s-2}\left\|v_{r}\right\|_{s} \\
& \leq C h^{-2}\left\|\left(R_{h} v\right)_{r}-v_{r}\right\|+C h^{-2}\left\|R_{h}\left(v_{r}\right)-v_{r}\right\|+C h^{s-2}\left\|v_{r}\right\|_{s} \\
& \leq C h^{-2} h^{s}\left\{\left\|v_{r}\right\|_{s}+\|v\|_{s}\right\}+C h^{-2} h^{s}\left\|v_{r}\right\|_{s}+C h^{s-2}\left\|v_{r}\right\|_{s} \\
& \leq C h^{s-2}\left\{\left\|v_{r}\right\|_{s}+\|v\|_{s}\right\} .
\end{aligned}
$$

The next theorem estimates the numerical error of the semidiscrete scheme in the $H^{1}(\mathcal{D})$ norm.

Theorem 3.7. Let $U$ be the solution of (1.8), $\tau=4$ (for example cubic splines approximation), $U \in H^{s}(\mathcal{D}$ ) and $U_{h}$ be the solution of the semidiscrete scheme (3.3). Under the assumptions (1.5) the next estimate holds, for $2 \leq s \leq \tau$

$$
\left\|U_{h}-U\right\|_{1} \leq\left\|U_{h}\left(R_{0}\right)-U\left(R_{0}\right)\right\|_{1}+C h^{s-\frac{3}{2}}+C h^{s-1}\left\|U\left(R_{0}\right)\right\|_{s} .
$$

Proof. Obviously since

$$
\left\|U_{h}-U\right\|_{1}=\|\varepsilon\|_{1}=\|\theta+\omega(U)\|_{1} \leq\|\theta\|_{1}+\|\omega(U)\|_{1},
$$

in order to estimate the error in the $H^{1}$-norm, we must provide a bound for $\|\theta\|_{1}$.

We subtract the weak formulation (2.5) and the semidiscrete scheme (3.3) to obtain for any $\phi \in S_{h} \subset$ $\widetilde{H}_{0}^{1}(\mathcal{D}) \cap H^{2}(\mathcal{D})$

$$
\begin{aligned}
& \left(\varepsilon_{r}-R_{h}\left(z \frac{s_{r}}{s} U_{h z}+\mathrm{i} \beta U_{h}\right)+z \frac{s_{r}}{s} U_{z}+\mathrm{i} \beta U, \phi\right)=-\mathrm{i} \tilde{a} \mathcal{B}(\varepsilon, \phi) \\
& +\mathrm{i} \tilde{a} \int_{z=1}\left\{\frac{s_{r}}{s}\left[\varepsilon_{r}-R_{h}\left(z \frac{s_{r}}{s} U_{h z}\right)+\frac{s_{r}}{s} U_{z}\right]-\frac{1}{s} \gamma(r, \vartheta) \varepsilon\right\} \bar{\phi} \mathrm{d} \vartheta .
\end{aligned}
$$

Recall the definition (2.4) of the form $\mathcal{B}$.

Here, and for the rest of this paper, the symbol $\omega$ whenever appears without input denotes $\omega(U)=R_{h}(U)-U$.

We choose now

$$
\phi:=\theta_{r}-R_{h}\left(z \frac{s_{r}}{s} \theta_{z}+\mathrm{i} \beta \theta\right) \in S_{h}
$$

and so, denoting

$$
\sigma:=R_{h}\left(z \frac{s_{r}}{s} \theta_{z}+\mathrm{i} \beta \theta\right)
$$

we have

$$
\phi=\theta_{r}-\sigma
$$


Remind that $\omega(u):=R_{h}(u)-u$ is well defined if $u \in H^{1}(\mathcal{D})$. The quantity

$$
\omega\left(z \frac{s_{r}}{s} \omega_{z}+\mathrm{i} \beta \omega\right) \equiv \omega\left(z \frac{s_{r}}{s} \omega_{z}(U)+\mathrm{i} \beta \omega(U)\right)
$$

(i.e. $\omega$ on $\left.z \frac{s_{r}}{s} \omega_{z}(U)+\mathrm{i} \beta \omega(U)\right)$ is well defined also since $R_{h} U \in S_{h} \subseteq \widetilde{H}_{0}^{1}(\mathcal{D}) \cap H^{2}(\mathcal{D})$, and furthermore $z \frac{s_{r}}{s} \omega_{z} \in \widetilde{H}_{0}^{1}(\mathcal{D})$.

In (3.16) we replace $\varepsilon$ by $\theta+\omega$. By the definition of $R_{h}$, we have $\mathcal{B}(\omega, \phi)=0$. So, we replace

$$
\phi=\theta_{r}-\sigma,
$$

we take imaginary parts and obtain after straightforward but extensive calculations, cf. [5],

$$
\begin{aligned}
& \operatorname{Re} \mathcal{B}\left(\theta, \theta_{r}\right)-\operatorname{Re} \mathcal{B}\left(\theta, z \frac{s_{r}}{s} \theta_{z}+\mathrm{i} \beta \theta\right)-[\tilde{A}+C \tilde{B}\|\theta\|+C \tilde{C}\|\sigma\|] \\
& \leq C \int_{z=1}\left\{\left|R_{h}(\mathrm{i} \beta \theta)\right|^{2}+\left|R_{h}\left(z \frac{s_{r}}{s} \omega_{z}\right)\right|^{2}+\left|\omega\left(z \frac{s_{r}}{s} U_{z}\right)\right|^{2}+\left|\omega_{r}-\frac{1}{s} \gamma(r, \vartheta) \omega\right|^{2}+|\gamma(r, \vartheta) \theta|^{2}\right\} \mathrm{d} \vartheta,
\end{aligned}
$$

for

$$
\begin{aligned}
\tilde{A} & :=\frac{\mathrm{d}}{\mathrm{d} r} \frac{1}{\tilde{a}} \operatorname{Im}\left(\left\{\omega\left(z \frac{s_{r}}{s} \omega_{z}+\mathrm{i} \beta \omega\right)+z \frac{s_{r}}{s} \omega_{z}+\mathrm{i} \beta \omega+\omega\left(z \frac{s_{r}}{s} U_{z}\right)+\omega(\mathrm{i} \beta U)-\omega_{r}\right\}, \theta\right), \\
\tilde{B} & :=\left\|\omega_{r}\left(z \frac{s_{r}}{s} \omega_{z}+\mathrm{i} \beta \omega\right)+\frac{\mathrm{d}}{\mathrm{d} r}\left[z \frac{s_{r}}{s} \omega_{z}+\mathrm{i} \beta \omega\right]+\omega_{r}\left(z \frac{s_{r}}{s} U_{z}\right)+\omega_{r}(\mathrm{i} \beta U)-\omega_{r r}\right\|, \\
\tilde{C} & :=\left\|\omega\left(z \frac{s_{r}}{s} \omega_{z}+\mathrm{i} \beta \omega\right)+z \frac{s_{r}}{s} \omega_{z}+\mathrm{i} \beta \omega+\omega\left(z \frac{s_{r}}{s} U_{z}\right)+\omega(\mathrm{i} \beta U)-\omega_{r}\right\| .
\end{aligned}
$$

Lemmas 2.2-2.4, yield

$$
\operatorname{Re} \mathcal{B}\left(\theta, \theta_{r}\right) \geq \frac{1}{2} \frac{\mathrm{d}}{\mathrm{d} r} \mathcal{B}(\theta, \theta)-C\|\theta\|_{1}^{2}
$$

and

$$
\operatorname{Re} \mathcal{B}\left(\theta, z \frac{s_{r}}{s} \theta_{z}+\mathrm{i} \beta \theta\right) \leq C\|\theta\|_{1}^{2} .
$$

Hence, (3.17) becomes the following main inequality

$$
\begin{aligned}
\frac{1}{2} \frac{\mathrm{d}}{\mathrm{d} r} \mathcal{B}(\theta, \theta) \leq & {[\tilde{A}+C \tilde{B}\|\theta\|+C \tilde{C}\|\sigma\|]+C\|\theta\|_{1}^{2} } \\
& +C \int_{z=1}\left\{\left|R_{h}(\mathrm{i} \beta \theta)\right|^{2}+\left|R_{h}\left(z \frac{s_{r}}{s} \omega_{z}\right)\right|^{2}+\left|\omega\left(z \frac{s_{r}}{s} U_{z}\right)\right|^{2}+\left|\omega_{r}-\frac{1}{s} \gamma(r, \vartheta) \omega\right|^{2}+|\gamma(r, \vartheta) \theta|^{2}\right\} \mathrm{d} \vartheta
\end{aligned}
$$

The inequality (3.18) is crucial for the proof, but as it contains many terms, we shall estimate each one of them in successive steps.

\section{Step 1:}

We shall proceed by estimating $\tilde{B}$.

Considering $\tilde{B}$, we will show that is bounded under the regularity assumption for $S_{h}$. Indeed, since $z \frac{s_{r}}{s} \omega_{z}, \mathrm{i} \beta \omega \in \widetilde{H}_{0}^{1}(\mathcal{D})$ then by Lemmas 3.6 and 3.5 , we get

$$
\begin{aligned}
\left\|\omega_{r}\left(z \frac{s_{r}}{s} \omega_{z}+\mathrm{i} \beta \omega\right)\right\| & \leq C h\left\{\left\|\omega_{z}\right\|_{1}+\left\|\omega_{z r}\right\|_{1}+\|\omega\|_{1}\right\} \leq C h\left\{\|\omega\|_{2}+\left\|\omega_{r}\right\|_{2}\right\} \\
& \leq C h h^{s-2}\left\{\|U\|_{s}+\left\|v_{r}\right\|_{s}\right\}=C h^{s-1}\left\{\|U\|_{s}+\left\|U_{r}\right\|_{s}\right\} .
\end{aligned}
$$


Also, we have

$$
\begin{aligned}
\left\|\frac{\mathrm{d}}{\mathrm{d} r}\left[z \frac{s_{r}}{s} \omega_{z}+\mathrm{i} \beta \omega\right]\right\| & \leq C\left\{\left\|\omega_{z}\right\|+\left\|\omega_{r z}\right\|+\|\omega\|+\left\|\omega_{r}\right\|\right\} \\
& \leq C\left\{\|\omega\|_{1}+\left\|\omega_{r}\right\|_{1}+\|\omega\|+\left\|\omega_{r}\right\|\right\} \\
& \leq C h^{s-1}\left\{\|U\|_{s}+\left\|U_{r}\right\|_{s}\right\},
\end{aligned}
$$

and

$$
\begin{aligned}
\left\|\omega_{r}\left(z \frac{s_{r}}{s} U_{z}\right)+\omega_{r}(\mathrm{i} \beta U)+\omega_{r r}\right\| & \leq C\left\{\left\|\omega_{r}\left(U_{z}\right)\right\|+\left\|\omega_{r}\right\|+\left\|\omega_{r r}\right\|\right\} \\
& \leq C h^{s-1}\left\{\|U\|_{s}+\left\|U_{r}\right\|_{s}+\left\|U_{r r}\right\|_{s}\right\} .
\end{aligned}
$$

Thus, we obtain

$$
\tilde{B} \leq C h^{s-1}\left\{\|U\|_{s}+\left\|U_{r}\right\|_{s}+\left\|U_{r r}\right\|_{s}\right\}
$$

\section{Step 2:}

Considering $\tilde{C}$ we have

$$
\begin{aligned}
\left\|\omega\left(z \frac{s_{r}}{s} \omega_{z}+\mathrm{i} \beta \omega\right)\right\| & \leq C\left\|\omega\left(\omega_{z}\right)+\omega(\omega)\right\| \leq C h\left\{\left\|\omega_{z}\right\|_{1}+\|\omega\|_{1}\right\} \\
& \leq C h\|\omega\|_{2} \leq C h^{s-1}\|U\|_{s},
\end{aligned}
$$

while the next inequality holds true

$$
\begin{aligned}
& \left\|z \frac{s_{r}}{s} \omega_{z}+\mathrm{i} \beta \omega+\omega\left(z \frac{s_{r}}{s} U_{z}\right)+\omega(\mathrm{i} \beta U)-\omega_{r}\right\| \\
& \leq C\left\{\|\omega\|_{1}+\|\omega\|+\left\|\omega\left(U_{z}\right)\right\|+\|\omega(U)\|+\left\|\omega_{r}\right\|\right\} \\
& \leq C h^{s-1}\|U\|_{s}+C h^{s}\left\|U_{z}\right\|_{s}+C h^{s}\left\{\|U\|_{s}+\left\|U_{r}\right\|_{s}\right\} \\
& \leq C h^{s-1}\left\{\|U\|_{s}+\left\|U_{r}\right\|_{s}\right\} .
\end{aligned}
$$

So, we get

$$
\tilde{C} \leq C h^{s-1}\left\{\|U\|_{s}+\left\|U_{r}\right\|_{s}\right\} .
$$

\section{Step 3:}

We replace the estimates of $\tilde{B}$ and $\tilde{C}$ in main inequality (3.17).

Indeed, using (3.19), (3.20), relation (3.17) is written as

$$
\begin{aligned}
& \frac{1}{2} \frac{\mathrm{d}}{\mathrm{d} r} \mathcal{B}(\theta, \theta) \leq \tilde{A}+C\|\theta\|_{1}^{2} \\
& +C h^{s-1}\left\{\|U\|_{s}+\left\|U_{r}\right\|_{s}+\left\|U_{r r}\right\|_{s}\right\}\|\theta\|+C h^{s-1}\left\{\|U\|_{s}+\left\|U_{r}\right\|_{s}\right\}\|\sigma\| \\
& +C \int_{z=1}\left\{\left|R_{h}(\mathrm{i} \beta \theta)\right|^{2}+\left|R_{h}\left(z \frac{s_{r}}{s} \omega_{z}\right)\right|^{2}+\left|\omega\left(z \frac{s_{r}}{s} U_{z}\right)\right|^{2}+\left|\omega_{r}-\frac{1}{s} \gamma(r, \vartheta) \omega\right|^{2}+|\gamma(r, \vartheta) \theta|^{2}\right\} \mathrm{d} \vartheta .
\end{aligned}
$$

Let us comment how we shall treat relation (3.21). The trace term and $\|\sigma\|$ will be suitably bounded. Furthermore, we shall integrate the resulting inequality. 


\section{Step 4:}

Considering the terms of the trace integral we first obtain

$$
\begin{aligned}
\int_{z=1}\left|R_{h}(\mathrm{i} \beta \theta)\right|^{2} \mathrm{~d} \vartheta & \leq C \int_{z=1}\left\{|\omega(\mathrm{i} \beta \theta)|^{2}+|\theta|^{2}\right\} \mathrm{d} \vartheta \\
& \leq C\|\omega(\theta)\|_{1}^{2}+C\|\theta\|_{1}^{2} \leq C\|\theta\|_{1}^{2}+C\|\theta\|_{1}^{2} \leq C\|\theta\|_{1}^{2} .
\end{aligned}
$$

The following term gives the lowest order in our estimates. In fact, using the inverse and trace inequalities, we arrive at

$$
\begin{aligned}
\int_{z=1}\left|R_{h}\left(z \frac{s_{r}}{s} \omega_{z}\right)\right|^{2} \mathrm{~d} \vartheta & \leq C\left\|R_{h}\left(z \frac{s_{r}}{s} \omega_{z}\right)\right\| \cdot\left\|R_{h}\left(z \frac{s_{r}}{s} \omega_{z}\right)\right\|_{1} \\
& \leq C h^{-1}\left\|R_{h}\left(z \frac{s_{r}}{s} \omega_{z}\right)\right\|^{2} \\
& \leq C h^{-1}\left\{\left\|\omega\left(z \frac{s_{r}}{s} \omega_{z}\right)\right\|^{2}+\left\|\omega_{z}\right\|^{2}\right\} \\
& \leq C h^{-1}\left(h^{2}\left\|\omega_{z}\right\|_{1}^{2}+h^{2 s-2}\|U\|_{s}^{2}\right) \\
& \leq C h^{-1}\left(h^{2} h^{2 s-4}\|U\|_{s}^{2}+h^{2 s-2}\|U\|_{s}^{2}\right) \\
& \leq C h^{2 s-3}\|U\|_{s}^{2} .
\end{aligned}
$$

Furthermore, the next relations hold true

$$
\begin{aligned}
& \int_{z=1}\left|\omega\left(z \frac{s_{r}}{s} U_{z}\right)\right|^{2} \mathrm{~d} \vartheta \leq C\left\|\omega\left(U_{z}\right)\right\|_{1}\left\|\omega\left(U_{z}\right)\right\| \leq C h^{2 s-3}\|U\|_{s}^{2} \\
& \int_{z=1}\left|\omega_{r}-\frac{1}{s} \gamma(r, \vartheta) \omega\right|^{2} \mathrm{~d} \vartheta \leq C h^{2 s-1}\left\{\|U\|_{s}^{2}+\left\|U_{r}\right\|_{s}^{2}\right\} \\
& \int_{z=1}|\gamma(r, \vartheta) \theta|^{2} \mathrm{~d} \vartheta \leq C\|\theta\|_{1}^{2} .
\end{aligned}
$$

\section{Step 5:}

We replace the estimates of trace integrals.

Indeed, using (3.22)-(3.24), relation (3.21) becomes

$$
\begin{gathered}
\frac{1}{2} \frac{\mathrm{d}}{\mathrm{d} r} \mathcal{B}(\theta, \theta) \leq \tilde{A}+C h^{s-1}\left\{\|U\|_{s}+\left\|U_{r}\right\|_{s}+\left\|U_{r r}\right\|_{s}\right\}\|\theta\|+C h^{s-1}\left\{\|U\|_{s}+\left\|U_{r}\right\|_{s}\right\}\|\sigma\| \\
+C h^{2 s-3}\|U\|_{s}^{2}+C h^{2 s-1}\left\{\|U\|_{s}^{2}+\left\|U_{r}\right\|_{s}^{2}\right\}+C\|\theta\|_{1}^{2} .
\end{gathered}
$$

\section{Step 6:}

We estimate $\|\sigma\|$ and replace in (3.25).

The inverse inequality gives

$$
\begin{aligned}
\|\sigma\| & =\left\|R_{h}\left(z \frac{s_{r}}{s} \theta_{z}+\mathrm{i} \beta \theta\right)\right\| \\
& \leq\left\|\omega\left(z \frac{s_{r}}{s} \theta_{z}+\mathrm{i} \beta \theta\right)\right\|+c\left\|\theta_{z}\right\|+C\|\theta\| \\
& \leq C h\left\|\theta_{z}\right\|_{1}+C h\|\theta\|_{1}+C\|\theta\|_{1} \\
& \leq C h\|\theta\|_{2} \leq C\|\theta\|_{1} .
\end{aligned}
$$


Thus, (3.25) takes the form

$$
\begin{aligned}
\frac{\mathrm{d}}{\mathrm{d} r} \mathcal{B}(\theta, \theta) \leq & 2 \tilde{A}+c h^{s-1}\left\{\|U\|_{s}+\left\|U_{r}\right\|_{s}+\left\|U_{r r}\right\|_{s}\right\}\|\theta\|_{1} \\
& +C\|\theta\|_{1}^{2}+C h^{2 s-3}\left(\|U\|_{s}^{2}+\left\|U_{r}\right\|_{s}^{2}\right) \\
\leq & 2 \tilde{A}+c h^{2 s-2}\left\{\|U\|_{s}^{2}+\left\|U_{r}\right\|_{s}^{2}+\left\|U_{r r}\right\|_{s}^{2}\right\} \\
& +C\|\theta\|_{1}^{2}+C h^{2 s-3}\left(\|U\|_{s}^{2}+\left\|U_{r}\right\|_{s}^{2}\right) \\
\leq & 2 \tilde{A}+C\|\theta\|_{1}^{2}+C h^{2 s-3}\left(\|U\|_{s}^{2}+\left\|U_{r}\right\|_{s}^{2}+\left\|U_{r r}\right\|_{s}^{2}\right) .
\end{aligned}
$$

We integrate (3.26) and obtain

$$
\begin{aligned}
\mathcal{B}(\theta, \theta) \leq & 2 \int_{R_{0}}^{r} \tilde{A} \mathrm{~d} s+C h^{2 s-3} \int_{R_{0}}^{r}\left[\|U\|_{s}^{2}+\left\|U_{r}\right\|_{s}^{2}+\left\|U_{r r}\right\|_{s}^{2}\right] \mathrm{d} s \\
& +C \int_{R_{0}}^{r}\|\theta\|_{1}^{2} \mathrm{~d} s+\mathcal{B}\left(R_{0} ; \theta, \theta\right) .
\end{aligned}
$$

\section{Step 7:}

We estimate the integral of $\tilde{A}$.

Easily, it follows that

$$
\begin{aligned}
\int_{R_{0}}^{r} \tilde{A} \mathrm{~d} s \leq & C\left\{\left\|\omega\left(\omega_{z}\right)\right\|+\|\omega(\omega)\|+\left\|\omega_{z}\right\|+\|\omega\|+\left\|\omega\left(U_{z}\right)\right\|+\left\|\omega_{r}\right\|\right\}\|\theta\|_{1} \\
& +C\left\{\left\|\omega\left(\omega_{z}\right)\right\|+\|\omega(\omega)\|+\left\|\omega_{z}\right\|+\|\omega\|+\left\|\omega\left(U_{z}\right)\right\|+\left\|\omega_{r}\right\|\right\}\left(R_{0}\right)\left\|\theta\left(R_{0}\right)\right\|_{1},
\end{aligned}
$$

where the notation $\{\cdots\}\left(R_{0}\right)$ denotes the quantities in these brackets computed at $r=R_{0}$. In addition, we have

$$
\begin{aligned}
& \left\|\omega\left(\omega_{z}\right)\right\| \leq C h\left\|\omega_{z}\right\|_{1} \leq C h h^{s-2}\|U\|_{s} \leq C h^{s-1}\|U\|_{s}, \\
& \|\omega(\omega)\| \leq C h\|\omega\|_{1} \leq C h h^{s-1}\|U\|_{s} \leq C h^{s}\|U\|_{s}, \\
& \left\|\omega\left(U_{z}\right)\right\| \leq C h^{s}\left\|U_{z}\right\|_{s} \leq C h^{s-1}\|U\|_{s} .
\end{aligned}
$$

In (3.28), we use the estimates for $\|\omega\|,\left\|\omega_{r}\right\|,\|\omega\|_{1}$ and (3.29). So, we obtain

$$
\int_{R_{0}}^{r} \tilde{A} \mathrm{~d} s \leq C h^{s-1}\left(\|U\|_{s}+\left\|U_{r}\right\|_{s}\right)\|\theta\|_{1}+C h^{s-1}\left(\left\|U\left(R_{0}\right)\right\|_{s}+\left\|U_{r}\left(R_{0}\right)\right\|_{s}\right)\left\|\theta\left(R_{0}\right)\right\|_{1} .
$$

\section{Final Step:}

Relations (3.27), (3.30) and Lemma 2.2 yield

$$
\begin{aligned}
C\|\theta\|_{1}^{2} \leq & \mathcal{B}(\theta, \theta) \\
\leq & C h^{2 s-2}\left(\|U\|_{s}+\left\|U_{r}\right\|_{s}\right)^{2}+C h^{2 s-3} \int_{R_{0}}^{r}\left[\|U\|_{s}^{2}+\left\|U_{r}\right\|_{s}^{2}+\left\|U_{r r}\right\|_{s}^{2}\right] \mathrm{d} s \\
& +C \int_{R_{0}}^{r}\|\theta\|_{1}^{2} \mathrm{~d} s+C h^{2 s-2}\left(\left\|U\left(R_{0}\right)\right\|_{s}+\left\|U_{r}\left(R_{0}\right)\right\|_{s}\right)^{2}+C\left\|\theta\left(R_{0}\right)\right\|_{1}^{2} .
\end{aligned}
$$

We must estimate first

$$
\delta(r):=\int_{R_{0}}^{r}\|\theta\|_{1}^{2} \mathrm{~d} s
$$


Using this notation, (3.31) is written as

$$
\frac{\mathrm{d}}{\mathrm{d} r} \delta(r) \leq \mathcal{E}+C \delta(r)
$$

for

$$
\begin{aligned}
\mathcal{E}:= & C h^{2 s-2}\left(\|U\|_{s}+\left\|U_{r}\right\|_{s}\right)^{2}+C h^{2 s-3} \int_{R_{0}}^{r}\left[\|U\|_{s}^{2}+\left\|U_{r}\right\|_{s}^{2}+\left\|U_{r r}\right\|_{s}^{2}\right] \mathrm{d} s \\
& +C h^{2 s-2}\left(\left\|U\left(R_{0}\right)\right\|_{s}+\left\|U_{r}\left(R_{0}\right)\right\|_{s}\right)^{2}+C\left\|\theta\left(R_{0}\right)\right\|_{1}^{2} .
\end{aligned}
$$

Integrating (3.32) yields

$$
\delta(r) \leq C \delta\left(R_{0}\right)+C \mathcal{E}=C \mathcal{E},
$$

i.e.

$$
\int_{R_{0}}^{r}\|\theta\|_{1}^{2} \mathrm{~d} s \leq C \mathcal{E}
$$

We replace in (3.31) this estimate, and we arrive at

$$
\|\theta\|_{1}^{2} \leq C h^{2 s-3}+C\left\|\theta\left(R_{0}\right)\right\|_{1}^{2} .
$$

By (3.33) and (3.14) the $H^{1}$ error estimate follows.

Observing the proof of Theorem 3.7 we easily see that when $s$ is only depending on the azimuth $\vartheta\left(s_{r}=0\right.$ for any $r, \vartheta)$ then the suboptimal term $\int_{z=1}\left|R_{h}\left(z \frac{s_{r}}{s} \omega_{z}\right)\right|^{2} \mathrm{~d} \vartheta$ vanishes. Furthermore, the results of all the presented lemmas and theorems hold true in this case also, $c f$. [5]. So, we obtain the next theorem that gives an optimal error in the $H^{1}(\mathcal{D})$ norm.

Theorem 3.8. Let $U$ be the solution of (1.8), $\tau=4$ (for example cubic splines approximation), $U \in H^{s}(\mathcal{D})$ and $U_{h}$ be the solution of the semidiscrete scheme (3.3). Under the assumptions (1.5), the next estimate holds true, for $2 \leq s \leq \tau$ and $r \in\left[R_{0}, R\right]$

$$
\left\|U_{h}-U\right\|_{1} \leq\left\|U_{h}\left(R_{0}\right)-U\left(R_{0}\right)\right\|_{1}+C h^{s-1}+C h^{s-1}\left\|U\left(R_{0}\right)\right\|_{s}
$$

\section{Crank-Nicolson fully Discrete schemes in RANGe}

We consider a uniform partition in the range interval $\left[R_{0}, R\right]$. More specifically, for $N \in \mathbb{N}^{*}$ we define $k:=\left(R-R_{0}\right) / N$ and $r^{n}:=R_{0}+n k$ for any $n=0, \ldots, N$. Further, let $r^{n+1 / 2}:=\left(r^{n}+r^{n+1}\right) / 2$ for any $n=0, \ldots, N-1$.

For $U^{n}$ given, we seek $U^{n+1} \in S_{h}$ such that

$$
\begin{aligned}
& \left(\frac{U^{n+1}-U^{n}}{k}-R_{h}\left(z \frac{s_{r}\left(r^{n+1 / 2}\right)}{s\left(r^{n+1 / 2}\right)}\left(\frac{U^{n+1}+U^{n}}{2}\right)_{z}+\mathrm{i} \beta\left(r^{n+1 / 2}\right) \frac{U^{n+1}+U^{n}}{2}\right), \phi\right)= \\
& -\mathrm{i} \tilde{a} \mathcal{B}\left(r^{n+1 / 2} ; \frac{U^{n+1}+U^{n}}{2}, \phi\right)+\left(f\left(r^{n+1 / 2}\right), \phi\right) \\
& +\mathrm{i} \tilde{a} \int_{z=1}\left[\frac{s_{r}\left(r^{n+1 / 2}\right)}{s\left(r^{n+1 / 2}\right)} R_{h}\left(\frac{U^{n+1}-U^{n}}{k}-z \frac{s_{r}\left(r^{n+1 / 2}\right)}{s\left(r^{n+1 / 2}\right)}\left(\frac{U^{n+1}+U^{n}}{2}\right)_{z}\right)-\frac{1}{s\left(r^{n+1 / 2}\right)} \gamma\left(r^{n+1 / 2}\right) \frac{U^{n+1}+U^{n}}{2}\right] \bar{\phi} \mathrm{d} \vartheta
\end{aligned}
$$

for any $\phi \in S_{h}, 0 \leq n \leq N-1$.

Here, $U^{0}$ is an appropriate projection of $U_{0}$ in $S_{\mathrm{h}}$. Moreover, the projection operator $R_{h}$ used is induced by $\mathcal{B}\left(r^{n+1 / 2} ; u, v\right)$. 
Remark 4.1. Obviously the fully discrete scheme is linear and is written in implicit form. In every step it demands the successive solution of 3 linear systems. More specifically, for $U^{n}$ given, (4.1) is implemented as follows:

1. Compute $R_{h}\left(z \frac{s_{r}\left(r^{n+1 / 2}\right)}{s\left(r^{n+1 / 2}\right)}\left(U^{n}\right)_{z}\right)$ by solving the linear system

$$
\mathcal{B}\left(r^{n+1 / 2} ; R_{h}\left(z \frac{s_{r}\left(r^{n+1 / 2}\right)}{s\left(r^{n+1 / 2}\right)}\left(U^{n}\right)_{z}\right), \phi\right)=\mathcal{B}\left(r^{n+1 / 2} ; z \frac{s_{r}\left(r^{n+1 / 2}\right)}{s\left(r^{n+1 / 2}\right)}\left(U^{n}\right)_{z}, \phi\right),
$$

for any $\phi$ in the basis of $S_{h}(N \times N$ 1st linear system which has a unique solution since $\mathcal{B}$ is well defined $)$. 2. Compute $R_{h}\left(\beta\left(r^{n+1 / 2}\right) U^{n}\right)$ by solving the linear system

$$
\mathcal{B}\left(r^{n+1 / 2} ; R_{h}\left(\beta\left(r^{n+1 / 2}\right) U^{n}\right), \phi\right)=\mathcal{B}\left(r^{n+1 / 2} ; \beta\left(r^{n+1 / 2}\right) U^{n}, \phi\right),
$$

for any $\phi$ in the basis of $S_{h}(N \times N$ 2nd linear system which has a unique solution since $\mathcal{B}$ is well defined). 3. Solve the following $3 N \times 3 N$ 3rd linear system for any $\phi$ in the basis of $S_{h}$

(4.1) (where the computed $R_{h}\left(z \frac{s_{r}\left(r^{n+1 / 2}\right)}{s\left(r^{n+1 / 2}\right)}\left(U^{n}\right)_{z}\right), R_{h}\left(\beta\left(r^{n+1 / 2}\right) U^{n}\right)$

are replaced)

$$
\begin{aligned}
& \mathcal{B}\left(r^{n+1 / 2} ; R_{h}\left(z \frac{s_{r}\left(r^{n+1 / 2}\right)}{s\left(r^{n+1 / 2}\right)}\left(U^{n+1}\right)_{z}\right), \phi\right)=\mathcal{B}\left(r^{n+1 / 2} ; z \frac{s_{r}\left(r^{n+1 / 2}\right)}{s\left(r^{n+1 / 2}\right)}\left(U^{n+1}\right)_{z}, \phi\right) \\
& \mathcal{B}\left(r^{n+1 / 2} ; R_{h}\left(\beta\left(r^{n+1 / 2}\right) U^{n+1}\right), \phi\right)=\mathcal{B}\left(r^{n+1 / 2} ; \beta\left(r^{n+1 / 2}\right) U^{n+1}, \phi\right) .
\end{aligned}
$$

Thus, the $3 N$ unknowns are:

$$
U^{n+1}, R_{h}\left(z \frac{s_{r}\left(r^{n+1 / 2}\right)}{s\left(r^{n+1 / 2}\right)}\left(U^{n+1}\right)_{z}\right) \text { and } R_{h}\left(\beta\left(r^{n+1 / 2}\right) U^{n+1}\right) \text {. }
$$

Remark 4.2. The numerical solution of these three systems which are solved successively results in an algorithm of numerical cost equivalent to solving a $5 N \times 5 N$ linear multi-diagonal system. Therefore, the fact that our scheme is implicit does not affect the numerical complexity of the algorithm.

On the other hand, the numerical implementation of the scheme is highly non-trivial. The complexity of simulations is due mainly to the following reasons: a) the spatial domain is 2-dimensional, b) the presence of a Neumann dynamical condition in 2 dimensions results in a cubic splines approximation which is of third polynomial order, (c) the Neumann condition involves a dynamical term which changes in every step in $r$. A forthcoming paper will be devoted to the numerical experiments and the discussion on the implementation issues.

Considering the 1-dimensional case with Neumann condition and for the 2-dimensional case with the modified Robin condition in place of the Neumann one, numerical simulations in piece-wise linear finite element spaces have been constructed by Antonopoulou in her Ph.D. thesis in [5]; cf. also in Ph.D. thesis of Sturm [17], and in $[8,9]$.

Now, by $H^{1}$ stability, we shall prove that the fully discrete scheme is well posed.

Proposition 4.3. Under the assumptions (1.5), the fully discrete scheme (4.1) is $H^{1}$-stable and admits a unique solution in $S_{h}$.

Proof. In (4.1) we take $f=0$.

We note that the non-homogeneous discrete problem which is given in implicit form is linear, so uniqueness which is equivalent to existence it is sufficient to be proven for $f=0$, i.e. for the homogeneous case. In addition, the stability argument in fact refers to the difference of two possible solutions that each of them satisfy the same fully discrete scheme (4.1) with different initial conditions. So, due to linearity, this difference satisfy the 
problem (4.1) with $f=0$ (homogeneous) and $U^{0}$ the resulting difference of initial conditions of the discrete problems.

We set

$$
\phi:=\frac{U^{n+1}-U^{n}}{k}-R_{h}\left(z \frac{s_{r}\left(r^{n+1 / 2}\right)}{s\left(r^{n+1 / 2}\right)}\left(\frac{U^{n+1}+U^{n}}{2}\right)_{z}+\mathrm{i} \beta\left(r^{n+1 / 2}\right) \frac{U^{n+1}+U^{n}}{2}\right),
$$

and get

$$
\begin{aligned}
& \|\phi\|^{2}=-\mathrm{i} \tilde{a} \mathcal{B}\left(r^{n+1 / 2} ; \frac{U^{n+1}+U^{n}}{2}, \phi\right) \\
& +\mathrm{i} \tilde{a} \int_{z=1}\left[\frac{s_{r}\left(r^{n+1 / 2}\right)}{s\left(r^{n+1 / 2}\right)} R_{h}\left(\frac{U^{n+1}-U^{n}}{k}-z \frac{s_{r}\left(r^{n+1 / 2}\right)}{s\left(r^{n+1 / 2}\right)}\left(\frac{U^{n+1}+U^{n}}{2}\right)\right)_{z}-\frac{1}{s\left(r^{n+1 / 2}\right)} \gamma\left(r^{n+1 / 2}\right) \frac{U^{n+1}+U^{n}}{2}\right] \bar{\phi} \mathrm{d} \vartheta .
\end{aligned}
$$

Observe now that

$$
\begin{aligned}
-\operatorname{Re} \mathcal{B}\left(r^{n+1 / 2} ; \frac{U^{n+1}+U^{n}}{2}, \phi\right)= & -\operatorname{Re} \mathcal{B}\left(r^{n+1 / 2} ; \frac{U^{n+1}+U^{n}}{2}, \frac{U^{n+1}-U^{n}}{k}\right) \\
& +\operatorname{Re} \mathcal{B}\left(r^{n+1 / 2} ; \frac{U^{n+1}+U^{n}}{2}, z \frac{s_{r}\left(r^{n+1 / 2}\right)}{s\left(r^{n+1 / 2}\right)}\left(\frac{U^{n+1}+U^{n}}{2}\right)_{z}\right) \\
& +\operatorname{Re} \mathcal{B}\left(r^{n+1 / 2} ; \frac{U^{n+1}+U^{n}}{2}, \mathrm{i} \beta\left(r^{n+1 / 2}\right) \frac{U^{n+1}+U^{n}}{2}\right) .
\end{aligned}
$$

We proceed by estimating the trace integral. The specific choice of $\phi$ is crucial, since the non-positive trace integral

$$
\operatorname{Re} \int_{z=1} \frac{s_{r}}{s}\left|\frac{U^{n+1}-U^{n}}{k}-R_{h}\left(z \frac{s_{r}}{s}\left(\frac{U^{n+1}+U^{n}}{2}\right)_{z}\right)\right|^{2} \mathrm{~d} \vartheta
$$

will appear. Indeed, as $s_{r} \leq 0$ it follows

$$
\begin{aligned}
& \operatorname{Re} \int_{z=1}\left[\frac{s_{r}}{s} R_{h}\left(\frac{U^{n+1}-U^{n}}{k}-z \frac{s_{r}}{s}\left(\frac{U^{n+1}+U^{n}}{2}\right)_{z}\right)-\frac{1}{s} \gamma \frac{U^{n+1}+U^{n}}{2}\right] \bar{\phi} \mathrm{d} \vartheta \\
= & \operatorname{Re} \int_{z=1}\left[\frac{s_{r}}{s} \frac{U^{n+1}-U^{n}}{k}-R_{h}\left(z \frac{s_{r}}{s}\left(\frac{U^{n+1}+U^{n}}{2}\right)\right) \bar{\phi} \mathrm{d} \vartheta\right. \\
& -\operatorname{Re} \int_{z=1} \frac{s_{r}}{s}\left(\frac{1}{s} \gamma \frac{U^{n+1}+U^{n}}{2}\right) \bar{\phi} \mathrm{d} \vartheta \\
= & \operatorname{Re} \int_{z=1} \frac{s_{r}}{s}\left|\frac{U^{n+1}-U^{n}}{k}-R_{h}\left(z \frac{s_{r}}{s}\left(\frac{U^{n+1}+U^{n}}{2}\right)_{z}\right)\right|^{2} \mathrm{~d} \vartheta \\
& \left.-\operatorname{Re} \int_{z=1} \frac{s_{r}}{s}\left(\frac{U^{n+1}-U^{n}}{k}-R_{h}\left(z \frac{s_{r}}{s}\left(\frac{U^{n+1}+U^{n}}{2}\right)\right)_{z}\right)\right) R_{h}\left(\mathrm{i} \beta \frac{U^{n+1}+U^{n}}{2}\right) \mathrm{d} \vartheta \\
& -\operatorname{Re} \int_{z=1} \frac{s_{r}}{s}\left(\frac{1}{s} \gamma \frac{U^{n+1}+U^{n}}{2}\right) \frac{U^{n+1}-U^{n}}{k}-R_{h}\left(z \frac{s_{r}}{s}\left(\frac{U^{n+1}+U^{n}}{2}\right)\right) \mathrm{d} \vartheta \\
& +\operatorname{Re} \int_{z=1} \frac{s_{r}}{s}\left(\frac{1}{s} \gamma \frac{U^{n+1}+U^{n}}{2}\right) \frac{R_{h}\left(\mathrm{i} \beta \frac{U^{n+1}+U^{n}}{2}\right) \mathrm{d} \vartheta}{\leq} \int_{z=1}\left\{\left|\frac{U^{n+1}+U^{n}}{2}\right|^{2}+\left|R_{h}\left(\mathrm{i} \beta \frac{U^{n+1}+U^{n}}{2}\right)\right|^{2}\right\} \mathrm{d} \vartheta \\
\leq & C \mid \frac{U^{n+1}+U^{n}}{2}\left\|_{1}^{2}+C\right\| R_{h}\left(\frac{U^{n+1}+U^{n}}{2}\right)\left\|_{1}^{2} \leq C\right\| \frac{U^{n+1}+U^{n}}{2} \|_{1}^{2} \cdot
\end{aligned}
$$


Note that for the above we used also the trace inequality together with relation (3.10).

Furthermore, in (4.2) we take imaginary parts and divide by $\tilde{a}$. Then we use relations (4.3), (4.4). So, we obtain

$$
\begin{aligned}
\operatorname{Re} \mathcal{B}\left(r^{n+1 / 2} ; \frac{U^{n+1}+U^{n}}{2}, \frac{U^{n+1}-U^{n}}{k}\right) \leq & \operatorname{Re} \mathcal{B}\left(r^{n+1 / 2} ; \frac{U^{n+1}+U^{n}}{2}, z \frac{s_{r}}{s}\left(\frac{U^{n+1}+U^{n}}{2}\right)_{z}\right) \\
& +C\left\|\frac{U^{n+1}+U^{n}}{2}\right\|_{1}^{2} \\
\leq & C\left\|\frac{U^{n+1}+U^{n}}{2}\right\|_{1}^{2},
\end{aligned}
$$

where for the last inequality we used Lemma 2.4.

In addition, it holds that

$$
\operatorname{Re} \mathcal{B}\left(r^{n+1 / 2} ; \frac{U^{n+1}+U^{n}}{2}, \frac{U^{n+1}-U^{n}}{k}\right)=\frac{1}{2 k}\left\{\mathcal{B}\left(r^{n+1 / 2} ; U^{n+1}, U^{n+1}\right)-\mathcal{B}\left(r^{n+1 / 2} ; U^{n}, U^{n}\right)\right\}
$$

So, we have by (4.5)

$$
\mathcal{B}\left(r^{n+1 / 2} ; U^{n+1}, U^{n+1}\right) \leq \mathcal{B}\left(r^{n+1 / 2} ; U^{n}, U^{n}\right)+C k\left\|U^{n+1}\right\|_{1}^{2}+C k\left\|U^{n}\right\|_{1}^{2},
$$

which yields

$$
\begin{aligned}
\mathcal{B}\left(r^{n+1} ; U^{n+1}, U^{n+1}\right) \leq & \mathcal{B}\left(r^{n} ; U^{n}, U^{n}\right)+\left[\mathcal{B}\left(r^{n+1 / 2} ; U^{n}, U^{n}\right)-\mathcal{B}\left(r^{n} ; U^{n}, U^{n}\right)\right] \\
& -\left[\mathcal{B}\left(r^{n+1 / 2} ; U^{n+1}, U^{n+1}\right)-\mathcal{B}\left(r^{n+1} ; U^{n+1}, U^{n+1}\right)\right] \\
& +C k\left\|U^{n+1}\right\|_{1}^{2}+C k\left\|U^{n}\right\|_{1}^{2} \\
\leq & \mathcal{B}\left(r^{n} ; U^{n}, U^{n}\right)+C k\left\|U^{n+1}\right\|_{1}^{2}+C k\left\|U^{n}\right\|_{1}^{2} .
\end{aligned}
$$

Here, we used the definition of $\mathcal{B}(r ; u, w)$ which is related to an $H^{1}$-type projection operator containing only terms of the form $\left(g(r) u_{a}, w_{b}\right)$ for $a, b=z, \vartheta$ and so

$$
\left|\mathcal{B}\left(r_{1} ; u, w\right)-\mathcal{B}\left(r_{2} ; u, w\right)\right| \leq C\left|r_{1}-r_{2}\right|\|u\|_{1}\|w\|_{1} .
$$

Thus, we obtain

$$
\begin{aligned}
\mathcal{B}\left(r^{n+1} ; U^{n+1}, U^{n+1}\right) & \leq \mathcal{B}\left(r^{n} ; U^{n}, U^{n}\right)+C k\left\|U^{n+1}\right\|_{1}^{2}+C k\left\|U^{n}\right\|_{1}^{2} \\
& \leq \mathcal{B}\left(r^{n} ; U^{n}, U^{n}\right)+C k \mathcal{B}\left(r^{n+1} ; U^{n+1}, U^{n+1}\right)+C k \mathcal{B}\left(r^{n} ; U^{n}, U^{n}\right),
\end{aligned}
$$

where we used relation (2.6). So, for $k$ small we arrive at

$$
\mathcal{B}\left(U^{n+1}, U^{n+1}\right) \leq\left(\frac{1+c k}{1-c k}\right) \mathcal{B}\left(U^{n}, U^{n}\right) .
$$

By (4.6) and (2.6) and since $k$ is small we get

$$
C_{1}\left\|U^{n+1}\right\|_{1} \leq \mathcal{B}\left(U^{n+1}, U^{n+1}\right) \leq C \mathcal{B}\left(U^{0}, U^{0}\right) \leq C\left\|U^{0}\right\|_{1} \text { (stability-uniqueness), }
$$

and therefore,

$$
\left\|U^{n+1}\right\| \leq C\left\|U^{0}\right\|_{1} .
$$

Hence, by uniqueness of solution we obtain existence. 


\subsection{Error estimates}

We define the error $\varepsilon^{n}:=U^{n}-U\left(r^{n}\right)$. We set

$$
\varepsilon^{n}=\theta^{n}+\omega^{n},
$$

with

$$
\theta^{n}=U^{n}-R_{h}^{n}\left(U\left(r^{n}\right)\right), \quad \text { and } \quad \omega^{n}:=R_{h}^{n}\left(U\left(r^{n}\right)\right)-U\left(r^{n}\right) .
$$

Let

$$
\Omega^{n}:=R_{h}^{n}\left(U\left(r^{n}\right)\right), \quad \text { and } \quad \Omega^{n+1 / 2}=\left(\Omega^{n+1}+\Omega^{n}\right) / 2,
$$

then

$$
U^{n}=\theta^{n}+\Omega^{n}
$$

Here, $R_{h}^{n}$ is induced by $\mathcal{B}\left(r^{n} ; u, v\right)$ so that

$$
\mathcal{B}\left(r^{n} ; R_{h}^{n}\left(U\left(r^{n}\right)\right), \phi\right)=\mathcal{B}\left(r^{n} ; U\left(r^{n}\right), \phi\right),
$$

for any $\phi \in S_{h}$. We also define

$$
\theta^{n+1 / 2}:=\left(\theta^{n+1}+\theta^{n}\right) / 2 .
$$

\subsubsection{Preliminaries}

The scheme (4.1) gives that for any $\phi \in S_{h}$

$$
\begin{aligned}
& \left(\frac{\theta^{n+1}-\theta^{n}+\Omega^{n+1}-\Omega^{n}}{k}-R_{h}\left(z \frac{s_{r}}{s}\left(\theta_{z}^{n+1 / 2}+\Omega_{z}^{n+1 / 2}\right)+\mathrm{i} \beta\left(\theta^{n+1 / 2}+\Omega^{n+1 / 2}\right)\right), \phi\right)= \\
& -\mathrm{i} \tilde{a} \mathcal{B}\left(r^{n+1 / 2} ; \theta^{n+1 / 2}+\Omega^{n+1 / 2}, \phi\right)+(f, \phi) \\
& +\mathrm{i} \tilde{a} \int_{z=1}\left\{\frac{s_{r}}{s} R_{h}\left(\frac{\theta^{n+1}-\theta^{n}+\Omega^{n+1}-\Omega^{n}}{k}-z \frac{s_{r}}{s}\left(\theta_{z}^{n+1 / 2}+\Omega_{z}^{n+1 / 2}\right)\right)\right. \\
& \left.-\frac{1}{s} \gamma\left(\theta^{n+1 / 2}+\Omega^{n+1 / 2}\right)\right\} \bar{\phi} \mathrm{d} \vartheta
\end{aligned}
$$

where $s, s_{r}, \beta, \gamma$ and $f$ are computed at $r:=r^{n+1 / 2}$. In the above, we use the identity

$$
\mathcal{B}\left(r^{n+1 / 2} ; \Omega^{n+1 / 2}, \phi\right)=\mathcal{B}\left(r^{n+1 / 2} ; \frac{U\left(r^{n+1}\right)+U\left(r^{n}\right)}{2}, \phi\right)+\mathcal{B}\left(r^{n+1 / 2}, E_{0}^{n}, \phi\right),
$$

for

$$
\begin{aligned}
E_{0}^{n}:= & \frac{1}{2}\left(\left[R_{h}^{n}\left(U\left(r^{n}\right)\right)-U\left(r^{n}\right)\right]+\left[R_{h}^{n+1}\left(U\left(r^{n+1}\right)\right)-U\left(r^{n+1}\right)\right]\right. \\
& \left.-\left[R_{h}^{n+1 / 2}\left(U\left(r^{n}\right)+U\left(r^{n+1}\right)\right)-\left(U\left(r^{n}\right)+U\left(r^{n+1}\right)\right)\right]\right),
\end{aligned}
$$


where $R_{h}^{n+1 / 2}$ is induced by $\mathcal{B}\left(r^{n+1 / 2} ; u, v\right)$. So, we obtain

$$
\begin{aligned}
& \left(\frac{\theta^{n+1}-\theta^{n}}{k}-R_{h}\left(z \frac{s_{r}}{s} \theta_{z}^{n+1 / 2}+\mathrm{i} \beta \theta^{n+1 / 2}\right), \phi\right)=-\mathrm{i} \tilde{a} \mathcal{B}\left(r^{n+1 / 2} ; \theta^{n+1 / 2}, \phi\right) \\
& +\mathrm{i} \tilde{a} \int_{z=1} \frac{s_{r}}{s}\left[\frac{\theta^{n+1}-\theta^{n}}{k}-R_{h}\left(z \frac{s_{r}}{s} \theta_{z}^{n+1 / 2}\right)\right] \bar{\phi} \mathrm{d} \vartheta-\mathrm{i} \tilde{a} \int_{z=1} \frac{1}{s} \gamma \theta^{n+1 / 2} \bar{\phi} \mathrm{d} \vartheta \\
& +\left(R_{h}\left(z \frac{s_{r}}{s} \Omega_{z}^{n+1 / 2}\right), \phi\right)+\left(R_{h}\left(\mathrm{i} \beta \Omega^{n+1 / 2}\right), \phi\right) \\
& -\mathrm{i} \tilde{a} \mathcal{B}\left(r^{n+1 / 2} ; \frac{U\left(r^{n+1}\right)+U\left(r^{n}\right)}{2}, \phi\right)+\mathrm{i} \tilde{a} \int_{z=1} \frac{s_{r}}{s} \frac{\Omega^{n+1}-\Omega^{n}}{k} \bar{\phi} \mathrm{d} \vartheta \\
& -\mathrm{i} \tilde{a} \int_{z=1} \frac{s_{r}}{s} R_{h}\left(z \frac{s_{r}}{s} \Omega_{z}^{n+1 / 2}\right) \bar{\phi} \mathrm{d} \vartheta-\mathrm{i} \tilde{a} \int_{z=1} \frac{1}{s} \gamma \Omega^{n+1 / 2} \bar{\phi} \mathrm{d} \vartheta \\
& -\left(\frac{\Omega^{n+1}-\Omega^{n}}{k}, \phi\right)+(f, \phi)-\mathrm{i} \tilde{a} \mathcal{B}\left(r^{n+1 / 2} ; E_{0}^{n}, \phi\right) .
\end{aligned}
$$

Observe that for the continuous problem it holds that

$$
\begin{aligned}
(f, \phi)= & \left(U_{r}-z \frac{s_{r}}{s} U_{z}-\mathrm{i} \beta U, \phi\right)+\mathrm{i} \tilde{a} \mathcal{B}\left(r^{n+1 / 2} ; U, \phi\right) \\
& -\mathrm{i} \tilde{a} \int_{z=1}\left\{\frac{s_{r}}{s}\left[U_{r}-\frac{s_{r}}{s} U_{z}\right]-\frac{1}{s} \gamma U\right\} \bar{\phi} \mathrm{d} \vartheta,
\end{aligned}
$$

for $f, s, s_{r}, \beta, \gamma$ and $U, U_{r}, U_{z}$ defined on $r:=r^{n+1 / 2}$ and $\phi$ is in $S_{h}$. In (4.8) we replace $(f, \phi)$ given by the previous formula and obtain

$$
\begin{aligned}
& \left(\frac{\theta^{n+1}-\theta^{n}}{k}-R_{h}\left(z \frac{s_{r}}{s} \theta_{z}^{n+1 / 2}+\mathrm{i} \beta \theta^{n+1 / 2}\right), \phi\right)= \\
& -\mathrm{i} \tilde{a} \mathcal{B}\left(r^{n+1 / 2} ; \theta^{n+1 / 2}, \phi\right)+\mathrm{i} \tilde{a} \int_{z=1} \frac{s_{r}}{s}\left[\frac{\theta^{n+1}-\theta^{n}}{k}-R_{h}\left(z \frac{s_{r}}{s} \theta_{z}^{n+1 / 2}\right)\right] \bar{\phi} \mathrm{d} \vartheta \\
& -\mathrm{i} \tilde{a} \int_{z=1} \frac{1}{s} \gamma \theta^{n+1 / 2} \bar{\phi} \mathrm{d} \vartheta+\left(E_{1}^{n}, \phi\right)+\left(E_{2}^{n}, \phi\right)+\left(E_{3}^{n}, \phi\right) \\
& +\mathrm{i} \tilde{a} \mathcal{B}\left(r^{n+1 / 2} ; E_{4}^{n}, \phi\right)-\mathrm{i} \tilde{a} \int_{z=1} \frac{s_{r}}{s} E_{1}^{n} \bar{\phi} \mathrm{d} \vartheta \\
& -\mathrm{i} \tilde{a} \int_{z=1} \frac{s_{r}}{s} E_{2}^{n} \bar{\phi} \mathrm{d} \vartheta+\mathrm{i} \tilde{a} \int_{z=1} \frac{1}{s} \gamma E_{5}^{n} \bar{\phi} \mathrm{d} \vartheta-\mathrm{i} \tilde{a} \mathcal{B}\left(r^{n+1 / 2} ; E_{0}^{n}, \phi\right),
\end{aligned}
$$

where

$$
\begin{aligned}
& E_{1}^{n}:=U_{r}\left(r^{n+1 / 2}\right)-\frac{\Omega^{n+1}-\Omega^{n}}{k}, \\
& E_{2}^{n}:=R_{h}\left(z \frac{s_{r}}{s} \Omega_{z}^{n+1 / 2}\right)-z \frac{s_{r}}{s} U_{z}\left(r^{n+1 / 2}\right), \\
& E_{3}^{n}:=R_{h}\left(\mathrm{i} \beta \Omega^{n+1 / 2}\right)-\mathrm{i} \beta U\left(r^{n+1 / 2}\right), \\
& E_{4}^{n}:=U\left(r^{n+1 / 2}\right)-\frac{U\left(r^{n+1}\right)+U\left(r^{n}\right)}{2} \\
& E_{5}^{n}:=U\left(r^{n+1 / 2}\right)-\Omega^{n+1 / 2}
\end{aligned}
$$

\subsubsection{Derivation of the fully discrete error estimate}

Analogously to the semidiscrete scheme, we choose

$$
\phi=\frac{\theta^{n+1}-\theta^{n}}{k}-R_{h}\left(z \frac{s_{r}\left(r^{n+1 / 2}\right)}{s\left(r^{n+1 / 2}\right)} \theta_{z}^{n+1 / 2}+\mathrm{i} \beta\left(r^{n+1 / 2}\right) \theta^{n+1 / 2}\right) .
$$


Then, we define

$$
\sigma^{n}:=R_{h}\left(z \frac{s_{r}\left(r^{n+1 / 2}\right)}{s\left(r^{n+1 / 2}\right)} \theta_{z}^{n+1 / 2}+\mathrm{i} \beta\left(r^{n+1 / 2}\right) \theta^{n+1 / 2}\right),
$$

and we write

$$
\phi=\frac{\theta^{n+1}-\theta^{n}}{k}-\sigma^{n} .
$$

In (4.9) we take imaginary parts and obtain

$$
\begin{aligned}
0= & -\tilde{a} \operatorname{Re} \mathcal{B}\left(r^{n+1 / 2} ; \theta^{n+1 / 2}, \phi\right)+\tilde{a} \int_{z=1} \frac{s_{r}}{s}|\phi|^{2} \mathrm{~d} \vartheta-\tilde{a} \operatorname{Re} \int_{z=1} \frac{1}{s} \gamma \theta^{n+1 / 2} \bar{\phi} \mathrm{d} \vartheta \\
& +\operatorname{Im}\left[\left(E_{1}^{n}, \phi\right)+\left(E_{2}^{n}, \phi\right)+\left(E_{3}^{n}, \phi\right)\right]+\tilde{a} \operatorname{Re} \mathcal{B}\left(r^{n+1 / 2} ; E_{4}^{n}, \phi\right) \\
& -\tilde{a} \operatorname{Re} \int_{z=1}\left[\frac{s_{r}}{s} E_{1}^{n}+\frac{s_{r}}{s} E_{2}^{n}-\frac{1}{s} \gamma E_{5}^{n}\right] \bar{\phi} \mathrm{d} \vartheta+\operatorname{Re} \int_{z=1} \frac{s_{r}}{s} R_{h}\left(\mathrm{i} \beta \theta^{n+1 / 2}\right) \bar{\phi} \mathrm{d} \vartheta \\
& -\tilde{a} \operatorname{Re} \mathcal{B}\left(r^{n+1 / 2} ; E_{0}^{n}, \phi\right) .
\end{aligned}
$$

In the above, we replace $\phi$ by (4.11) in the first sesquilinear form and in the inner products. Since $s_{r} \leq 0$ we use $\int_{z=1} \frac{s_{r}}{s}|\phi|^{2} \mathrm{~d} \vartheta$ to bound the trace integrals and then apply the trace inequality to obtain

$$
\begin{aligned}
& \operatorname{Re} \mathcal{B}\left(r^{n+1 / 2} ; \theta^{n+1 / 2}, \frac{\theta^{n+1}-\theta^{n}}{k}\right) \leq \operatorname{Re} \mathcal{B}\left(r^{n+1 / 2} ; \theta^{n+1 / 2}, \sigma^{n}\right) \\
& +\int_{z=1}\left|C_{0}\right|^{2} \frac{s_{r}}{s}|\phi|^{2} \mathrm{~d} \vartheta+C\left\|\theta^{n+1 / 2}\right\|_{1}^{2} \\
& +\frac{1}{\tilde{a}}\left[\left\|E_{1}^{n}\right\|+\left\|E_{2}^{n}\right\|+\left\|E_{3}^{n}\right\|\right]\left\|\sigma^{n}\right\|+\operatorname{Re} \mathcal{B}\left(r^{n+1 / 2} ; E_{4}^{n}, \phi\right) \\
& +\frac{1}{\tilde{a} k} \operatorname{Im}\left[\left(E_{1}^{n}, \theta^{n+1}-\theta^{n}\right)+\left(E_{2}^{n}, \theta^{n+1}-\theta^{n}\right)+\left(E_{3}^{n}, \theta^{n+1}-\theta^{n}\right)\right] \\
& +C \int_{z=1}\left(\left|E_{1}^{n}\right|^{2}+\left|E_{2}^{n}\right|^{2}+\left|E_{5}^{n}\right|^{2}\right) \mathrm{d} \vartheta-\operatorname{Re} \mathcal{B}\left(r^{n+1 / 2} ; E_{0}^{n}, \phi\right),
\end{aligned}
$$

where we used the relation (3.10).

The trace inequality applied on the trace integral of (4.12), gives

$$
\begin{aligned}
& \operatorname{Re} \mathcal{B}\left(r^{n+1 / 2} ; \theta^{n+1 / 2}, \theta^{n+1}-\theta^{n}\right) \leq k \operatorname{Re} \mathcal{B}\left(r^{n+1 / 2} ; \theta^{n+1 / 2}, \sigma^{n}\right) \\
& +k \int_{z=1}\left|C_{0}\right|^{2} \frac{s_{r}}{s}|\phi|^{2} \mathrm{~d} \vartheta+C k\left\|\theta^{n+1 / 2}\right\|_{1}^{2}+C k\left\{\left\|E_{1}^{n}\right\|+\left\|E_{2}^{n}\right\|+\left\|E_{3}^{n}\right\|\right\}\left\|\sigma^{n}\right\| \\
& +k \operatorname{Re} \mathcal{B}\left(r^{n+1 / 2} ; E_{4}^{n}-E_{0}^{n}, \phi\right) \\
& +C k\left\{\left\|E_{1}^{n}\right\|_{1}\left\|E_{1}^{n}\right\|+\left\|E_{2}^{n}\right\|_{1}\left\|E_{2}^{n}\right\|+\left\|E_{5}^{n}\right\|_{1}\left\|E_{5}^{n}\right\|\right\}+H^{n}
\end{aligned}
$$

where

$$
H^{n}:=\frac{1}{\tilde{a}} \operatorname{Im}\left(E_{1}^{n}+E_{2}^{n}+E_{3}^{n}, \theta^{n+1}-\theta^{n}\right) .
$$


We observe that if $\phi \in S_{h}$ then

$$
\begin{aligned}
& \mathcal{B}\left(r^{n+1 / 2} ; E_{4}^{n}-E_{0}^{n}, \phi\right)=-\left(\left[\frac{1}{s^{2}}\left\{1+z^{2} \frac{s_{\vartheta}^{2}}{r^{2}}\right\}\left(E_{4 z}^{n}-E_{0 z}^{n}\right)\right]_{z}, \phi\right) \\
& +\int_{z=1} \frac{1}{s^{2}}\left\{1+z^{2} \frac{s_{\vartheta}^{2}}{r^{2}}\right\}\left(E_{4 z}^{n}-E_{0 z}^{n}\right) \bar{\phi} \mathrm{d} \vartheta \\
& -\frac{1}{r^{2}}\left(E_{4 \vartheta \vartheta}^{n}-E_{0 \vartheta \vartheta}^{n}, \phi\right)+\frac{1}{r^{2}}\left(\left[z \frac{s_{r}}{s}\left(E_{4 z}^{n}-E_{0 z}^{n}\right)\right]_{\vartheta}, \phi\right) \\
& -\frac{1}{r^{2}}\left(\left[z \frac{s_{r}}{s}\left(E_{4 \vartheta}^{n}-E_{0 \vartheta}^{n}\right)\right]_{z}, \phi\right)+\int_{z=1} z \frac{s_{r}}{s}\left(E_{4 \vartheta}^{n}-E_{0 \vartheta}^{n}\right) \bar{\phi} \mathrm{d} \vartheta .
\end{aligned}
$$

In (4.14) we take real parts. Then for $\tilde{C}_{0}>0$ a constant as small as needed, and since

$$
E_{4}^{n}:=U\left(r^{n+1 / 2}\right)-\frac{U\left(r^{n+1}\right)+U\left(r^{n}\right)}{2}, E_{4 z}^{n} \leq C k^{2}, \quad E_{4 \vartheta}^{n} \leq C k^{2},
$$

we have, for $\left.\Lambda\right|_{z=1}$ of the form $\left.\left[\left(R_{h}^{m}-I\right)(g)\right]_{a}\right|_{z=1}$ with $a=z, \vartheta$

$$
\begin{aligned}
& k \operatorname{Re} \mathcal{B}\left(r^{n+1 / 2} ; E_{4}^{n}-E_{0}^{n}, \phi\right) \leq k\left(\mathcal{A}^{n}, \phi\right)+C k\left[\left\|E_{4 z}^{n}\right\|_{\infty}+\left\|E_{4 \vartheta}^{n}\right\|_{\infty}\right] \int_{z=1}|\phi| \mathrm{d} \vartheta \\
& +C k \int_{z=1}|\Lambda \| \phi| \mathrm{d} \vartheta \\
& \leq k\left(\mathcal{A}^{n}, \phi\right)+C k k^{2} \int_{z=1}|\phi| \mathrm{d} \vartheta+C \int_{z=1} k^{1 / 2}|\Lambda| k^{1 / 2}|\phi| \mathrm{d} \vartheta \\
& \leq k\left(\mathcal{A}^{n}, \phi\right)+C \int_{z=1} k^{5 / 2} k^{1 / 2}|\phi| \mathrm{d} \vartheta+C \int_{z=1} k^{1 / 2}|\Lambda| k^{1 / 2}|\phi| \mathrm{d} \vartheta \\
& \leq k\left(\mathcal{A}^{n}, \phi\right)+C k^{5}+\tilde{C}_{0} k \int_{z=1}|\phi|^{2} \mathrm{~d} \vartheta+C k \int_{z=1}|\Lambda|^{2} \mathrm{~d} \vartheta \\
& \leq C k\left\|E_{4}^{n}-E_{0}^{n}\right\|_{2}\left\|\sigma^{n}\right\|+\left(\mathcal{A}^{n}, \theta^{n+1}-\theta^{n}\right)+C k^{5}+\tilde{C}_{0} k \int_{z=1}|\phi|^{2} \mathrm{~d} \vartheta+C k\|\Lambda\|\|\Lambda\|_{1} \\
& \leq C k\left\|E_{4}^{n}-E_{0}^{n}\right\|_{2}\left\|\sigma^{n}\right\|+\left(\mathcal{A}^{n}, \theta^{n+1}-\theta^{n}\right)+C k^{5}+\tilde{C}_{0} k \int_{z=1}|\phi|^{2} \mathrm{~d} \vartheta+C k h^{s-1} h^{s-2} .
\end{aligned}
$$

Here, $\mathcal{A}^{n}$ are of the form:

$$
\begin{aligned}
& \mathcal{C}^{n} E^{n}, \\
& \mathcal{C}^{n}=g\left(r^{n+1 / 2}, z, \vartheta\right), \\
& E^{n}=E_{z}^{n}, E_{\vartheta}^{n}, E_{z z}^{n}, E_{z \vartheta}^{n}, E_{\vartheta \vartheta}^{n}, \quad \text { for } \quad E^{n}=E_{4}^{n}-E_{0}^{n} .
\end{aligned}
$$

In (4.13) we use the definition of $R_{h}$, relation (4.15), and the identity

$$
\operatorname{Re} \mathcal{B}\left(r^{n+1 / 2} ; \theta^{n+1 / 2}, \theta^{n+1}-\theta^{n}\right)=\frac{1}{2}\left[\mathcal{B}\left(r^{n+1 / 2} ; \theta^{n+1}, \theta^{n+1}\right)-\mathcal{B}\left(r^{n+1 / 2} ; \theta^{n}, \theta^{n}\right)\right] .
$$

So, under the assumptions on $s_{r}$ relation (4.13) gives finally

$$
\begin{aligned}
& \operatorname{Re} \mathcal{B}\left(r^{n+1 / 2} ; \theta^{n+1}, \theta^{n+1}\right) \leq \operatorname{Re} \mathcal{B}\left(r^{n+1 / 2} ; \theta^{n}, \theta^{n}\right) \\
& +2 k \mathcal{B}\left(r^{n+1 / 2} ; \theta^{n+1 / 2}, z \frac{s_{r}}{s} \theta_{z}^{n+1 / 2}+\mathrm{i} \beta \theta^{n+1 / 2}\right)+C k\left\|\theta^{n+1 / 2}\right\|_{1}^{2} \\
& +C k\left\{\left\|E_{1}^{n}\right\|+\left\|E_{2}^{n}\right\|+\left\|E_{3}^{n}\right\|\right\}\left\|\sigma^{n}\right\|+C k\left\|E_{4}^{n}-E_{0}^{n}\right\|_{2}\left\|\sigma^{n}\right\| \\
& +\left(\mathcal{A}^{n}, \theta^{n+1}-\theta^{n}\right)+C k^{5}+C k h^{2 s-3} \\
& +k\left\{\left\|E_{1}^{n}\right\|_{1}\left\|E_{1}^{n}\right\|+\left\|E_{2}^{n}\right\|_{1}\left\|E_{2}^{n}\right\|+\left\|E_{5}^{n}\right\|_{1}\left\|E_{5}^{n}\right\|\right\}+H^{n} .
\end{aligned}
$$


We set

$$
\begin{aligned}
Z^{n}:= & C k\left\{\left\|E_{1}^{n}\right\|+\left\|E_{2}^{n}\right\|+\left\|E_{3}^{n}\right\|\right\}\left\|\sigma^{n}\right\|+C k\left\|E_{4}^{n}-E_{0}^{n}\right\|_{2}\left\|\sigma^{n}\right\| \\
& +\left|\left(\mathcal{A}^{n}, \theta^{n+1}-\theta^{n}\right)\right|+C k^{5}+C k h^{2 s-3} \\
& +k\left\{\left\|E_{1}^{n}\right\|_{1}\left\|E_{1}^{n}\right\|+\left\|E_{2}^{n}\right\|_{1}\left\|E_{2}^{n}\right\|+\left\|E_{5}^{n}\right\|_{1}\left\|E_{5}^{n}\right\|\right\}+H^{n},
\end{aligned}
$$

then, using Lemma 2.4, relation (4.17) yields

$$
\operatorname{Re} \mathcal{B}\left(r^{n+1 / 2} ; \theta^{n+1}, \theta^{n+1}\right) \leq \operatorname{Re} \mathcal{B}\left(r^{n+1 / 2} ; \theta^{n}, \theta^{n}\right)+C k\left\|\theta^{n+1}\right\|_{1}^{2}+C k\left\|\theta^{n}\right\|_{1}^{2}+Z^{n} .
$$

This is the main inequality that we shall use to derive the error estimate.

\section{Step 1:}

Observe that (4.18), for $k$ sufficiently small, gives

$$
\operatorname{Re} \mathcal{B}\left(r^{n+1} ; \theta^{n+1}, \theta^{n+1}\right) \leq \frac{1+C k}{1-C k} \operatorname{Re} \mathcal{B}\left(r^{n} ; \theta^{n}, \theta^{n}\right)+\frac{1}{1-C k} Z^{n} .
$$

We apply now (4.19) for $i=1, \ldots, n$ and get

$$
\operatorname{Re} \mathcal{B}\left(r^{n+1} ; \theta^{n+1}, \theta^{n+1}\right) \leq C \operatorname{Re} \mathcal{B}\left(\theta^{0}, \theta^{0}\right)+C \sum_{i=0}^{n} Z^{n-i} .
$$

So, by relation (4.20) we arrive at

$$
\left\|\theta^{n+1}\right\|_{1}^{2} \leq C\left\|\theta^{0}\right\|_{1}^{2}+C \sum_{i=0}^{n} Z^{i}
$$

\section{Step 2:}

Our aim will be to estimate $\sum_{i=0}^{n} Z^{i}$.

For this, we will provide bounds for sums involving: $\left|\left(\mathcal{A}^{n}, \theta^{n+1}-\theta^{n}\right)\right|,\left\|E_{4}^{n}-E_{0}^{n}\right\|_{2},\left\|\sigma^{n}\right\|, H^{n}$, and $E_{i}^{n}$, $i=1, \ldots, 5$ in various norms.

For general $\mathcal{A}^{n}$, it holds that

$$
\left|\sum_{i=0}^{n}\left(\mathcal{A}^{i}, \theta^{i+1}-\theta^{i}\right)\right| \leq\left|\left(\mathcal{A}^{0}, \theta^{0}\right)\right|+\sum_{i=0}^{n}\left\|\mathcal{A}^{i}-\mathcal{A}^{i-1}\right\|\left\|\theta^{i}\right\|+\left|\left(\mathcal{A}^{n}, \theta^{n+1}\right)\right| .
$$

Further by the definition of $E_{4}^{i}, E_{0}^{i}$ we have that

$$
\left\|E_{4}^{i}-E_{0}^{i}\right\|_{2} \leq C k^{2}+C h^{s-2},
$$

while by Taylor expansion easily we have that

$$
\left\|\left[E_{4}^{i}-E_{0}^{i}\right]-\left[E_{4}^{i-1}-E_{0}^{i-1}\right]\right\|_{2}=\left\|\left[E_{4}^{i}-E_{4}^{i-1}\right]-\left[E_{0}^{i}-E_{0}^{i-1}\right]\right\|_{2} \leq C k^{3}+C k h^{s-2},
$$

and

$$
\left\|\mathcal{C}^{i}-\mathcal{C}^{i-1}\right\| \leq C k
$$

So, for the specific $\mathcal{A}^{n}$ given by (4.16), we get

$$
\begin{aligned}
\left\|\mathcal{A}^{i}-\mathcal{A}^{i-1}\right\| & =\left\|\mathcal{C}^{i} E^{i}-\mathcal{C}^{i-1} E^{i-1}\right\| \leq\left\|\mathcal{C}^{i}\left(E^{i}-E^{i-1}\right)\right\|+\left\|E^{i-1}\left(\mathcal{C}^{i}-\mathcal{C}^{i-1}\right)\right\| \\
& \leq C k^{3}+C k h^{s-2}+C\left(k^{2}+h^{s-2}\right) k \leq C k^{3}+C k h^{s-2} .
\end{aligned}
$$


Therefore, we have finally

$$
\left|\sum_{i=0}^{n}\left(\mathcal{A}^{i}, \theta^{i+1}-\theta^{i}\right)\right| \leq C\left(k^{2}+h^{s-2}\right)\left\|\theta^{0}\right\|+C\left(k^{3}+k h^{s-2}\right) \sum_{i=0}^{n}\left\|\theta^{i}\right\|+C\left(k^{2}+h^{s-2}\right)\left\|\theta^{n+1}\right\| .
$$

We proceed by proving the next lemma.

Lemma 4.4. For any $\sigma^{i}$ it holds that

$$
\left\|\sigma^{i}\right\| \leq C\left\|\theta^{i+1}\right\|_{1}+C\left\|\theta^{i}\right\|_{1} .
$$

Proof. By the inverse inequality in $S_{h}$ we arrive at

$$
\begin{aligned}
\left\|\sigma^{i}\right\| & \leq\left\|\left(R_{h}-I\right)\left(z \frac{s_{r}}{s} \theta_{z}^{i+1 / 2}+\mathrm{i} \beta \theta^{i+1 / 2}\right)\right\|+C\left\|\theta^{i+1 / 2}\right\|_{1} \\
& \leq C\left\|\theta^{i+1}\right\|_{1}+C\left\|\theta^{i}\right\|_{1} .
\end{aligned}
$$

Let us estimate now the sum of $H^{i}$.

Lemma 4.5. It holds that

$$
\begin{aligned}
\sum_{i=0}^{n} H^{i} \leq & C\left\|\theta^{0}\right\|_{1} \sum_{\rho=1}^{3}\left\|E_{\rho}^{0}\right\|+C\left\|\theta^{n+1}\right\|_{1} \sum_{\rho=1}^{3}\left\|E_{\rho}^{n}\right\| \\
& +\sum_{\rho=1}^{3} \sum_{i=1}^{n}\left\|E_{\rho}^{i}-E_{\rho}^{i-1}\right\|\left\|\theta^{i}\right\| .
\end{aligned}
$$

Proof. For any sequence $d^{i}$ of smooth complex functions, easily it follows that

$$
\left|\sum_{i=0}^{n}\left(d^{i}, \theta^{i+1}-\theta^{i}\right)\right| \leq\left|\left(d^{0}, \theta^{0}\right)\right|+\sum_{i=0}^{n}\left\|d^{i}-d^{i-1}\right\|\left\|\theta^{i}\right\|+\left|\left(d^{n}, \theta^{n+1}\right)\right|,
$$

while

$$
\left|\sum_{i=0}^{n} \mathcal{B}\left(d^{i}, \theta^{i+1}-\theta^{i}\right)\right| \leq\left|\mathcal{B}\left(d^{0}, \theta^{0}\right)\right|+\sum_{i=0}^{n}\left|\mathcal{B}\left(d^{i}-d^{i-1}, \theta^{i}\right)\right|+\left|\mathcal{B}\left(d^{n}, \theta^{n+1}\right)\right| .
$$

Applying the above estimates for $d^{i}:=E_{1}^{i}, d^{i}:=E_{2}^{i}$ and $d^{i}:=E_{3}^{i}$, we obtain the result.

By the definitions of $Z^{i}$ and $H^{i}$ and the previous lemma it is obvious that we must estimate the terms $E_{\rho}^{i}$ and $E_{\rho}^{i}-E_{\rho}^{i-1}$. This is done at the next lemma which follows from standard calculations.

Lemma 4.6. The next estimates hold true

$$
\begin{aligned}
& \left\|E_{\rho}^{i}-E_{\rho}^{i-1}\right\| \leq C k\left\{h^{s-1}+k^{2}\right\}, \quad \rho=1,2,3 \\
& \left\|E_{1}^{n}\right\| \leq C\left\{h^{s}+k^{2}\right\}, \quad\left\|E_{1}^{n}\right\|_{1} \leq C\left\{h^{s-1}+k^{2}\right\} \\
& \left\|E_{2}^{n}\right\| \leq C\left\{h^{s-1}+k^{2}\right\}, \quad\left\|E_{2}^{n}\right\|_{1} \leq C\left\{h^{s-2}+k^{2}\right\} \\
& \left\|E_{3}^{n}\right\| \leq C\left\{h^{s}+k^{2}\right\} \\
& \left\|E_{4}^{n}\right\|_{1} \leq C k^{2}, \quad\left\|E_{4}^{n}\right\|_{2} \leq C k^{2} \\
& \left\|E_{5}^{n}\right\| \leq C\left\{h^{s}+k^{2}\right\}, \quad\left\|E_{5}^{n}\right\|_{1} \leq C\left\{h^{s-1}+k^{2}\right\} .
\end{aligned}
$$

Note that the worst order term is $\left\|E_{2}^{n}\right\|_{1}$.

A bound for the sum of $Z^{i}$ is given at the next lemma. 
Lemma 4.7. For $\mathcal{M}:=\left\{h^{s-3 / 2}+k^{2}\right\}^{2}$, it holds that

$$
\sum_{i=0}^{n} Z^{i} \leq C\left[\left\{h^{s-2}+k^{2}\right\}\left\|\theta^{0}\right\|_{1}+\left\{h^{s-2}+k^{2}\right\}\left\|\theta^{n+1}\right\|_{1}+k\left\{h^{s-2}+k^{2}\right\} \sum_{i=0}^{n+1}\left\|\theta^{i}\right\|_{1}+\mathcal{M}\right] .
$$

Proof. Lemma 4.6 gives

$$
\begin{aligned}
& \left\|E_{1}^{i}\right\|+\left\|E_{2}^{i}\right\|+\left\|E_{3}^{i}\right\|+\left\|E_{4}^{i}-E_{0}^{i}\right\|_{2} \leq C\left\{h^{s-2}+k^{2}\right\} \\
& \left\|E_{1}^{i}\right\|_{1}\left\|E_{1}^{i}\right\|+\left\|E_{2}^{i}\right\|_{1}\left\|E_{2}^{i}\right\|+\left\|E_{5}^{i}\right\|_{1}\left\|E_{5}^{i}\right\| \leq C\left\{h^{2 s-3}+k^{4}\right\} .
\end{aligned}
$$

By Lemmas 4.5, 4.6, we obtain

$$
\sum_{i=0}^{n} H^{i} \leq C\left\{h^{s-1}+k^{2}\right\}\left\|\theta^{0}\right\|_{1}+C\left\{h^{s-1}+k^{2}\right\}\left\|\theta^{n+1}\right\|_{1}+C \sum_{i-1}^{n} k\left\{h^{s-1}+k^{2}\right\}\left\|\theta^{i}\right\|_{1} .
$$

Relations (4.25), (4.23), (4.26) and the estimate of $\left\|\sigma^{i}\right\|$ of Lemma 4.4 yield the result.

\section{Final Step:}

We replace the estimate $(4.24)$ in $(4.21)$.

Furthermore, we consider $h \leq C k$. This assumption is reasonable since we are in the far field of sound propagation in range ( $r>>0$, which is the evolutionary variable), while the transformed problem in space is posed on the small rectangle $\mathcal{D}=[0,1] \times\left[\vartheta_{1}, \vartheta_{2}\right]$. So, for $s=4$ we have

$$
\left\|\theta^{n+1}\right\|_{1}^{2} \leq C\left\|\theta^{0}\right\|_{1}^{2}+\frac{1}{2}\left\|\theta^{n+1}\right\|_{1}^{2}+\frac{1}{4} \max _{0 \leq i \leq n+1}\left\|\theta^{i}\right\|_{1}^{2}+G^{2},
$$

where $G:=C\left\{h^{s-3 / 2}+k^{2}\right\}$. Taking the maximum in $n$, relation (4.27) finally gives

$$
\left\|\theta^{n}\right\|_{1} \leq C\left\|\theta^{0}\right\|_{1}+C\left\{h^{s-3 / 2}+k^{2}\right\}
$$

Now we can prove the next theorem that estimates the error $\varepsilon^{n}=U^{n}-U\left(r^{n}\right)$ in the $H^{1}$ norm.

Theorem 4.8. Under the assumptions (1.5) (upsloping bottom), if $U$ the solution of $(1.8)$ with $U \in H^{s}(\mathcal{D})$ the next inequalities hold true:

1. If $h \leq C k$, then

$$
\left\|\varepsilon^{n}\right\|_{1} \leq C\left\|U^{0}-U\left(R_{0}\right)\right\|_{1}+C\left\{h^{s-3 / 2}+k^{2}\right\}
$$

for $s=\tau=4$.

2. If the bottom topography depends only on azimuth $\vartheta$, then

$$
\left\|\varepsilon^{n}\right\|_{1} \leq C\left\|U^{0}-U\left(R_{0}\right)\right\|_{1}+C\left\{h^{s-1}+k^{2}\right\}
$$

for any $2 \leq s \leq \tau=4$

Proof. By (4.28) and the estimates of $\omega$ we get the first inequality.

Easily (as in the semidiscrete estimate), when $s_{r}=0$ then the suboptimal term $\left\|E_{2}^{n}\right\|_{1}$ vanishes and we obtain the second optimal error estimate.

Acknowledgements. The main results of this paper appeared in my Ph.D. thesis. I would like to thank my Advisor Professor V. A. Dougalis and Professor G. E. Zouraris for the encouragement and advice offered. I would like also to thank the two anonymous referees for their valuable comments and suggestions. 


\section{REFERENCES}

[1] L. Abrahamsson and H.O. Kreiss, The initial boundary value problem for the Schrödinger equation. Math. Methods Appl. Sci. 13 (1990a) 385-390.

[2] L. Abrahamsson and H.O. Kreiss, Boundary conditions for the parabolic equation in a range-dependent duct. J. Acoust. Soc. Amer. 87 (1990b) 2438-2441.

[3] G.D. Akrivis and V.A. Dougalis, Finite difference discretization with variable mesh of the Schrödinger equation in a variable domain. Bull. Greek Math. Soc. 31 (1990) 19-28.

[4] G.D. Akrivis, V.A. Dougalis and G.E. Zouraris, Finite difference schemes for the 'Parabolic' Equation in a variable depth environment with a rigid bottom boundary condition. SIAM J. Numer. Anal. 39 (2001) 539-565.

[5] D.C. Antonopoulou, Theory and numerical analysis of parabolic approximations. Ph.D. thesis (in Greek). University of Athens, Greece (2006).

[6] D.C. Antonopoulou, Galerkin methods for the 'Parabolic Equation' Dirichlet problem in a variable 2-D and 3-D topography, Appl. Numer. Math. 67 (2013) 17-34.

[7] D.C. Antonopoulou and M. Plexousakis, Discontinuous Galerkin methods for the linear Schrödinger equation in noncylindrical domains. Numer. Math. 115 (2010) 585-608.

[8] D.C. Antonopoulou, V.A. Dougalis and G.E. Zouraris, Galerkin methods for parabolic and Schrödinger equations with dynamical boundary conditions and applications to underwater acoustics. SIAM J. Numer. Anal. 47 (2009) $2752-2781$.

[9] D.C. Antonopoulou, G.D. Karali, M. Plexousakis and G.E. Zouraris, Crank-Nicolson finite element discretizations for a two dimensional linear Schrödinger-type equation posed in a noncylindrical domain. Math. Comput. 84 (2015) 1571-1598.

[10] S.C. Brenner and L.R. Scott, The Mathematical Theory of Finite Element Methods. In vol. 15 of Texts Appl. Math. SpringerVerlag, New York (1994).

[11] V.A. Dougalis, N.A. Kampanis, F. Sturm, and G.E. Zouraris, Numerical solution of the Parabolic Equation in range-dependent waveguides in Effective Computational Methods for Wave Propagation. Edited by N.A. Kampanis et al. Chapman and Hall/CRC, Boca Raton (2008) 175-207.

[12] M. Ehrhardt, Discrete artificial boundary conditions. Ph.D. thesis, Technische Universität, Berlin (2001).

[13] J. Escher, Quasilinear parabolic systems with dynamical boundary conditions, Commun. Partial. Differ. Eqs. 18 (1993) $1309-1364$.

[14] L.C. Evans, Partial Differential Equations. In vol. 19 of Grad. Stud. Math. American Mathematical Society (1998).

[15] F.B. Jensen, W.A. Kuperman, M.B. Porter, H. Schmidt, Computational Ocean Acoustics. AIP Series in Modern Acoustics and Signal Processing. American Institute of Physics, New York (1994).

[16] M.H. Schultz, Spline Analysis. Prentice-Hall (1973).

[17] F. Sturm, Modélisation mathématique et numérique d' un problème de propagation en acoustique sous-marine: prise en compte d'un environnement variable tridimensionnel. Ph.D. thesis, Université de Toulon et du Var, France (1997).

[18] F.D. Tappert, The parabolic approximation method, in Wave Propagation and Underwater Acoustics, edited by J.B. Keller and J.S. Papadakis. In vol. 70 of Lect. Notes Phys. Springer-Verlag, Berlin (1977) 224-287.

[19] J.L. Vázquez and E. Vitillaro, Heat equation with dynamical boundary conditions of reactive type. Commun. Partial. Differ. Eqs. 33 (2008) 561-612. 